\title{
PLUG-IN ESTIMATION OF LEVEL SETS IN A NON-COMPACT SETTING WITH APPLICATIONS IN MULTIVARIATE RISK THEORY
}

\author{
Elena Di Bernardino ${ }^{1}$, Thomas Lalö̈ ${ }^{2}$, Véronique Maume-Deschamps ${ }^{1}$ \\ And ClÉmentine PRIEuR ${ }^{3}$
}

\begin{abstract}
This paper deals with the problem of estimating the level sets $L(c)=\{F(x) \geq c\}$, with $c \in(0,1)$, of an unknown distribution function $F$ on $\mathbb{R}_{+}^{2}$. A plug-in approach is followed. That is, given a consistent estimator $F_{n}$ of $F$, we estimate $L(c)$ by $L_{n}(c)=\left\{F_{n}(x) \geq c\right\}$. In our setting, noncompactness property is a priori required for the level sets to estimate. We state consistency results with respect to the Hausdorff distance and the volume of the symmetric difference. Our results are motivated by applications in multivariate risk theory. In particular we propose a new bivariate version of the conditional tail expectation by conditioning the two-dimensional random vector to be in the level set $L(c)$. We also present simulated and real examples which illustrate our theoretical results.
\end{abstract}

Mathematics Subject Classification. 62G05, 62G20, 60E05, 91B30.

Received March 22, 2011. Revised July 13, 2011.

\section{INTRODUCTION}

The conditional tail expectation (CTE) is a commonly used tool in the univariate risk theory. Given a random variable $X$ with continuous and strictly monotonic distribution function $F_{X}$, the CTE at level $\alpha$ is defined by

$$
\operatorname{CTE}_{\alpha}(X)=\mathbb{E}\left[X \mid X \geq Q_{X}(\alpha)\right]
$$

with $\alpha \in(0,1)$ and $Q_{X}(\alpha)$ the univariate quantile function of $X$ at level $\alpha$. In other words the $\operatorname{CTE}_{\alpha}(X)$ is defined by the expectation value of $X$ conditionally to the fact that $X$ belongs to a certain one-dimensional level set of $F_{X}$. For further details about this univariate risk measure we refer the reader e.g. to Denuit et al. [16].

In this paper we are interested to introduce a new bivariate generalization of CTE in (1.1) and to provide a consistent procedure to estimate it. Consequently we essentially need a generalization of the notion of quantile in dimension higher than one. Therefore we remark that the quantile function is a relevant tool in statistics and

\footnotetext{
Keywords and phrases. Level sets, distribution function, plug-in estimation, Hausdorff distance, conditional tail expectation.

1 Université de Lyon, Université Lyon 1, ISFA, Laboratoire SAF, 50 Avenue Tony Garnier, 69366 Lyon, France. elena.di-bernardino@univ-lyon1.fr; veronique.maume@univ-lyon1.fr

2 Université de Nice Sophia-Antipolis, Laboratoire J-A Dieudonné, Parc Valrose, 06108 Nice Cedex 02, France. thomas.laloe@unice.fr

3 Université Joseph Fourier, Tour IRMA, MOISE-LJK B.P. 5338041 Grenoble, France. clementine.prieur@imag.fr
} 
probability, but its practical use is mostly confined to the univariate case where it is easily interpretable and calculable. The bivariate (or multivariate) characterization has received some attention however, it is much less common, due to a number of theoretical and practical reasons. In particular the main difficulty is that there does not exist a natural ordering in $n$-dimensions, for $n>1$. For this reason, in the last decade several attempts have been made to provide a multidimensional generalization of the univariate quantile function. We refer to Serfling [31] for a complete review on the topic. For example Massé and Theodorescu [23] defined multivariate quantiles as halfplanes and Koltchinskii [21] provided a general treatment of multivariate quantiles as inversions of mappings.

As a possible generalization of the univariate quantile function, Tibiletti [32], Fernández-Ponce and SuárezLloréns [18] and Belzunce et al. [4] defined a multivariate quantile as a set of points which accumulate the same probability for a fixed orthant. They called it level curves or quantile curves. In this sense de Haan and Huang [14] model a risk-problem of flood in the bivariate setting using an estimator of level curves of bivariate distribution function. In this paper we will follow this approach in order to propose a new risk measure which generalizes the CTE in (1.1). To this end we need to estimate the level sets of a bivariate distribution function $F$. This leads us into the general field of level sets estimation.

The problem of estimating level sets of an unknown function (for instance of a density function and more recently a regression function) has received attention recently. In particular the estimation of density level sets has been studied in Polonik [25], Tsybakov [33], Baíllo et al. [3], Baíllo [2], Cadre [7], Rigollet and Vert [27], Biau et al. [5]. The estimation of regression level sets in a compact setting has been analyzed in Cavalier [9], Laloë [22]. An alternative approach, based on the geometric properties of the compact support sets, has been presented by Hartigan [20], Cuevas and Fraiman [11], Cuevas and Rodríguez-Casal [12]. The problem of estimating general level sets under compactness assumptions has been discussed by Cuevas et al. [13]. The asymptotic behavior of minimum volume sets and of a generalized quantile process is analyzed by Polonik [26].

Considering the level sets of a bivariate distribution function, the commonly assumed property of compactness for these sets is no more reasonable. Then, differing from the literature cited above, we need to work in a noncompact setting and this requires special attention in the statement of our problem.

Our general approach will be the following: first, we provide a consistent estimator of the level set

$$
L(c)=\{F(x) \geq c\}, \quad \text { for } c \in(0,1) .
$$

Therefore we consider a plug-in approach (e.g. see Baíllo et al. [3]; Rigollet and Vert [27]; Cuevas et al. [13]), that is, $L(c)$ is estimated by

$$
L_{n}(c)=\left\{F_{n}(x) \geq c\right\}, \quad \text { for } c \in(0,1),
$$

where $F_{n}$ is a consistent estimator of $F$. The regularity properties of $F$ and $F_{n}$ as well as the consistency properties of $F_{n}$ will be specified in the statements of our theorems.

In order to provide a consistency results we define two proximity criteria between sets. A standard choice is the volume of the symmetric difference. Another natural criterion is given by the Hausdorff distance that corresponds to an intuitive notion of "physical proximity" between sets. Our consistency properties are stated with respect to these two criteria under reasonable assumptions on $F$ and $F_{n}$ (Thms. 3.1-4.1). These results are based on a slight modification of Proposition 3.1 in the Ph.D. thesis of Rodríguez-Casal [28] (Prop. 2.1).

We then introduce our new bivariate version of the Conditional Tail Expectation by conditioning the twodimensional random vector to be in the level set $L(c)$. This new risk measure is based on the bivariate Valueat-Risk proposed in Embrechts and Puccetti [17] and Nappo and Spizzichino [24] and it is different from the existing bivariate generalizations of the univariate conditional tail expectation (e.g. see Cai and Li [8]). Indeed we do not use any aggregate risk in the portfolio in order to analyze the bivariate risk's issue. Our bivariate version of the CTE deals with the simultaneous joint damages considering the dependence structure of data in a bivariate specific risk's area $(L(c))$. We propose an estimator for this new risk measure using plug-in approach for level sets and provide a consistency result (Thm. 5.3). 
The paper is organized as follows. We introduce some notation, tools and technical assumptions in Section 2. Consistency and asymptotic properties of our estimator of $L(c)$ are given in Sections 3 and 4 . In Section 5 we introduce a new bivariate generalization of CTE in (1.1) and we provide a consistent estimator for this risk measure. Illustrations with simulated and real data are presented in Section 6 . Section 7 summarizes and briefly mentions directions for future research. Finally, some auxiliary results and more technical proofs are postponed to Section 8.

\section{NotATION AND PRELIMINARIES}

In this section we introduce some notation and tools which will be useful later.

Let $\mathbb{N}^{*}=\mathbb{N} \backslash\{0\}, \mathbb{R}_{+}^{2}=\mathbb{R}_{+}^{2} \backslash(0,0), \mathcal{F}$ the set of continuous distribution functions $\mathbb{R}_{+}^{2} \rightarrow[0,1]$ and $F \in \mathcal{F}$. Given an $i . i . d$ sample $\left\{X_{i}\right\}_{i=1}^{n}$ in $\mathbb{R}_{+}^{2}$ with distribution function $F$, we denote by $F_{n}(\cdot)=F_{n}\left(X_{1}, X_{2}, \ldots, X_{n}, \cdot\right)$ an estimator of $F$ based on the finite sample $\left(X_{1}, X_{2}, \ldots, X_{n}\right)$. We restrict ourselves to $\mathbb{R}_{+}^{2}$ for convenience but the following results are completely adaptable in $\mathbb{R}^{2}$. This choice is motivated essentially by our application in risk theory proposed in Section 5, where random variables will be losses then defined in a positive support.

Define, for $c \in(0,1)$, the upper c-level set of $F \in \mathcal{F}$ and its plug-in estimator

$$
L(c)=\left\{x \in \mathbb{R}_{+}^{2}: F(x) \geq c\right\}, \quad L_{n}(c)=\left\{x \in \mathbb{R}_{+}^{2}: F_{n}(x) \geq c\right\},
$$

and

$$
\{F=c\}=\left\{x \in \mathbb{R}_{+}^{2}: F(x)=c\right\}
$$

In addition, given $T>0$, we set

$$
\begin{gathered}
L(c)^{T}=\left\{x \in[0, T]^{2}: F(x) \geq c\right\}, L_{n}(c)^{T}=\left\{x \in[0, T]^{2}: F_{n}(x) \geq c\right\}, \\
\{F=c\}^{T}=\left\{x \in[0, T]^{2}: F(x)=c\right\} .
\end{gathered}
$$

Furthermore, for any $A \subset \mathbb{R}_{+}^{2}$ we denote by $\partial A$ its boundary.

Note that $\{F=c\}$ can be a portion of quadrant $\mathbb{R}_{+}^{2}$ instead of a set of Lebesgue measure null in $\mathbb{R}_{+}^{2}$ (that is the presence of plateau at level $c$ ). In the following we will introduce suitable conditions in order to avoid the presence of plateau in the graph of $F$.

In the metric space $\left(\mathbb{R}_{+}^{2}, d\right)$, where $d$ stands for the Euclidean distance, we denote by $B(x, \rho)$ the closed ball centered on $x$ and with positive radius $\rho$. Let $B(S, \rho)=\bigcup_{x \in S} B(x, \rho)$, with $S$ a closed set of $\mathbb{R}_{+}^{2}$. For $r>0$ and $\zeta>0$, define

$$
E=B\left(\left\{x \in \mathbb{R}_{+}^{2}:|F(x)-c| \leq r\right\}, \zeta\right),
$$

and, for a twice differentiable function $F$,

$$
m^{\nabla}=\inf _{x \in E}\left\|(\nabla F)_{x}\right\|, \quad M_{H}=\sup _{x \in E}\left\|(H F)_{x}\right\|,
$$

where $(\nabla F)_{x}$ is the gradient vector of $F$ evaluated at $x$ and $\left\|(\nabla F)_{x}\right\|$ its Euclidean norm, $(H F)_{x}$ the Hessian matrix evaluated in $x$ and $\left\|(H F)_{x}\right\|$ its matrix norm induced by the Euclidean norm.

For sake of completeness, we recall that if $A_{1}$ and $A_{2}$ are compact sets in $\left(\mathbb{R}_{+}^{2}, d\right)$, the Hausdorff distance between $A_{1}$ and $A_{2}$ is defined by

$$
d_{H}\left(A_{1}, A_{2}\right)=\inf \left\{\rho>0: A_{1} \subset B\left(A_{2}, \rho\right), A_{2} \subset B\left(A_{1}, \rho\right)\right\},
$$

or equivalently by

$$
d_{H}\left(A_{1}, A_{2}\right)=\max \left\{\sup _{x \in A_{1}} d\left(x, A_{2}\right), \sup _{x \in A_{2}} d\left(x, A_{1}\right)\right\},
$$

where $d\left(x, A_{2}\right)=\inf _{y \in A_{2}}\|x-y\|$. 

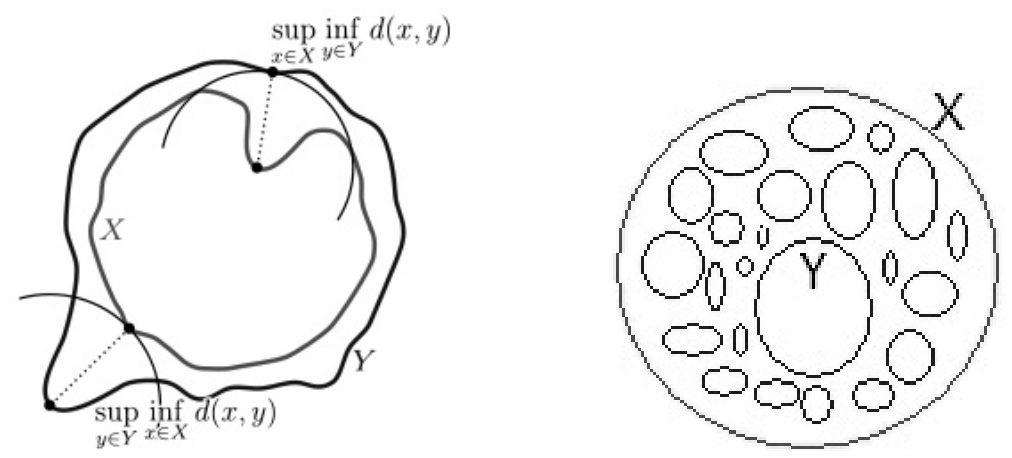

Figure 1. (left) Hausdorff distance between sets; (right) particular case where $d_{H}(X, Y)$ does not capture the shape properties of $X$ and $Y$.

The above expression is well-defined even when $A_{1}$ and $A_{2}$ are just closed (not necessarily compact) sets but in this case the value $d_{H}\left(A_{1}, A_{2}\right)$ could be infinity. Then in our setting, in order to avoid these situations we need a truncated version of Assumption $T$ in Cuevas et al. [13] or Tsybakov [33]. More precisely we introduce the following assumption.

H: There exist $\gamma>0$ and $A>0$ such that, if $|t-c| \leq \gamma$ then $\forall T>0$ such that $\{F=c\}^{T} \neq \emptyset$ and $\{F=t\}^{T} \neq \emptyset$

$$
d_{H}\left(\{F=c\}^{T},\{F=t\}^{T}\right) \leq A|t-c| .
$$

Assumption $\mathbf{H}$ is satisfied under mild conditions. Proposition 2.1 below is a slight modification of Proposition 3.1 in the Ph.D. thesis of Rodríguez-Casal [28] in order to deal with non-compact sets.

Proposition 2.1. Let $c \in(0,1)$. Let $F \in \mathcal{F}$ be twice differentiable on $\mathbb{R}_{+}^{2 *}$. Assume there exist $r>0, \zeta>0$ such that $m^{\nabla}>0$ and $M_{H}<\infty$. Then $F$ satisfies Assumption $\mathbf{H}$, with $A=\frac{2}{m^{\nabla}}$.

The proof is postponed to Section 8 .

Remark 2.2. Under assumptions of Proposition 2.1, $F$ is continuous and $m^{\nabla}>0$, there is no plateau in the graph of $F$ for each level $t$ such that $|t-c| \leq r$. Furthermore from Theorem 1 in Rossi [30] we know that each half-line in $\mathbb{R}_{+}^{2}$, parallel to one of the axis, meets $\{F=t\}$ in a connected set of points. As a consequence we obtain that $\{F=t\}$ is a curve in the quadrant $\mathbb{R}_{+}^{2}$. In particular, from $m^{\nabla}>0$, for a fixed $x$ we have to consider all corresponding values $y$ in a specific interval (we refer the interested reader to Rem. 2.1 in Rossi [30] or Rem. 3.1 in Rossi [29]). In this case the plane curve $\{F=t\}$ is not the graph of a function but it has the following monotonic property. We consider $(x, y),\left(x^{\prime}, y^{\prime}\right) \in\{F=t\}$, if $x<x^{\prime}$ then $y \geq y^{\prime}$, if $y<y^{\prime}$ then $x \geq x^{\prime}$. In particular if we suppose that each component of $(\nabla F)_{x}$ is greater than zero in $E$ then $\{F=t\}$ is a monotone decreasing curve in $\mathbb{R}_{+}^{2}$. Finally under assumptions of Proposition 2.1 we obtain $\partial L(c)^{T}=\{F=c\}^{T}=\{F=c\} \cap[0, T]^{2}$ (we refer for details to Thm. 3.2 in Rodríguez-Casal [28]).

\section{Consistency in terms of the HausdorfF Distance}

In this section we study the consistency properties of $L_{n}(c)^{T}$ with respect to the Hausdorff distance between $\partial L_{n}(c)^{T}$ and $\partial L(c)^{T}$. The Hausdorff distance corresponds to an intuitive notion of "physical proximity" between sets (see Fig. 1 (left) for a graphical illustration). However the metric $d_{H}$ is not always completely successful in capturing the shape properties: two sets can be very close in $d_{H}$ and still have quite different shapes. For instance in Figure 1 (right) we have $X$, the outside ball, and $Y$ the set of balls inside $X$. The distance $d_{H}(X, Y)$ is small even if $X$ and $Y$ are quite different in many important aspects. 
In order to avoid these situations, following Cuevas and Rodríguez-Casal [12] and Cuevas et al. [13], a way to reinforce the notion of visual proximity between two sets provided by $d_{H}$ is to impose the proximity of the respective boundaries. We will follow this approach in Theorem 3.1 below.

From now on we note, for $n \in \mathbb{N}^{*}$,

$$
\left\|F-F_{n}\right\|_{\infty}=\sup _{x \in \mathbb{R}_{+}^{2}}\left|F(x)-F_{n}(x)\right|
$$

and for $T>0$

$$
\left\|F-F_{n}\right\|_{\infty}^{T}=\sup _{x \in[0, T]^{2}}\left|F(x)-F_{n}(x)\right| .
$$

The following result can be considered an adapted version of Theorem 2 in Cuevas et al. [13].

Theorem 3.1. Let $c \in(0,1)$. Let $F \in \mathcal{F}$ be twice differentiable on $\mathbb{R}_{+}^{2 *}$. Assume that there exist $r>0, \zeta>0$ such that $m^{\nabla}>0$ and $M_{H}<\infty$. Let $T_{1}>0$ such that for all $t:|t-c| \leq r, \partial L(t)^{T_{1}} \neq \emptyset$. Let $\left(T_{n}\right)_{n \in \mathbb{N}^{*}}$ be an increasing sequence of positive values. Assume that, for each $n$ and for almost all samples of size $n, F_{n}$ is a continuous function and that

$$
\left\|F-F_{n}\right\|_{\infty} \rightarrow 0, \quad \text { a.s. }
$$

Then

$$
d_{H}\left(\partial L(c)^{T_{n}}, \partial L_{n}(c)^{T_{n}}\right)=O\left(\left\|F-F_{n}\right\|_{\infty}\right), \quad \text { a.s. }
$$

The proof is postponed to Section 8. Under assumptions of Theorem 3.1, $d_{H}\left(\partial L(c)^{T_{n}}, \partial L_{n}(c)^{T_{n}}\right)$ converges to zero and the quality of our plug-in estimator is obviously related to the quality of the estimator $F_{n}$.

Remark 3.2. Theorem 3.1 provides an asymptotic result for a fixed level $c$. In particular following the proof of Theorem 3.1 (postponed to Sect. 8) we remark that, for $n$ large enough,

$$
d_{H}\left(\partial L(c)^{T_{n}}, \partial L_{n}(c)^{T_{n}}\right) \leq 6 A\left\|F-F_{n}\right\|_{\infty}^{T_{n}}, \quad \text { a.s. },
$$

where $A=\frac{2}{m^{\nabla}}$. Note that in the case $c$ is close to one the constant $A$ could be large. In this case, we will need a large number of data to get a reasonable estimation.

Note that the empirical distribution estimator does not satisfy continuity assumption imposed in Theorem 3.1. However, in order to overcome this problem it can be considered a smooth version of this estimator (e.g. see Chaubey and Sen [10]), which satisfies convergence in (3.1). However we remark that the continuity assumption, imposed in Theorem 3.1, will not be necessary in the following results.

\section{4. $L_{1}$ CONSISTENCY}

The previous section was devoted to the consistency of $L_{n}$ in terms of the Hausdorff distance. We consider now another consistency criterion: the consistency of the volume (in the Lebesgue measure sense) of the symmetric difference between $L(c)^{T_{n}}$ and $L_{n}(c)^{T_{n}}$. This means that we define the distance between two subsets $A_{1}$ and $A_{2}$ of $\mathbb{R}_{2}^{+}$by

$$
d_{\lambda}\left(A_{1}, A_{2}\right)=\lambda\left(A_{1} \triangle A_{2}\right),
$$

where $\lambda$ stands for the Lebesgue measure on $\mathbb{R}^{2}$ and $\triangle$ for the symmetric difference.

Let us introduce the following assumption:

A1 There exist positive increasing sequences $\left(v_{n}\right)_{n \in \mathbb{N}^{*}}$ and $\left(T_{n}\right)_{n \in \mathbb{N}^{*}}$ such that

$$
v_{n} \int_{\left[0, T_{n}\right]^{2}}\left|F-F_{n}\right|^{p} \lambda(\mathrm{d} x) \underset{n \rightarrow \infty}{\stackrel{\mathbb{P}}{\rightarrow}} 0
$$

for some $1 \leq p<\infty$. 
We now establish our consistency result in terms of the volume of the symmetric difference. We can interpret the following theorem as a generalization of Theorem 3 in Cuevas et al. [13] (Sect. 3) in the case of noncompact level sets. In addition, we provide also a convergence rate for the symmetric difference between $L(c)^{T_{n}}$ and $L_{n}(c)^{T_{n}}$.

Theorem 4.1. Let $c \in(0,1)$. Let $F \in \mathcal{F}$ be twice differentiable on $\mathbb{R}_{+}^{2 *}$. Assume that there exist $r>0, \zeta>0$ such that $m^{\nabla}>0$ and $M_{H}<\infty$. Assume that for each $n$, with probability one, $F_{n}$ is measurable. Let $\left(v_{n}\right)_{n \in \mathbb{N}^{*}}$ and $\left(T_{n}\right)_{n \in \mathbb{N}^{*}}$ positive increasing sequences such that Assumption $\mathbf{A} \mathbf{1}$ is satisfied and that for all $t:|t-c| \leq r$, $\partial L(t)^{T_{1}} \neq \emptyset$. Then it holds that

$$
p_{n} d_{\lambda}\left(L(c)^{T_{n}}, L_{n}(c)^{T_{n}}\right) \underset{n \rightarrow \infty}{\stackrel{\mathbb{P}}{\rightarrow}} 0
$$

with $p_{n}$ an increasing positive sequence such that $p_{n}=o\left(v_{n}^{\frac{1}{p+1}} / T_{n}^{\frac{p}{p+1}}\right)$.

The proof is postponed to Section 8. Theorem 4.1 provides a convergence rate, which is closely related to the choice of the sequence $T_{n}$. A sequence $T_{n}$ whose divergence rate is large implies a convergence rate $p_{n}$ quite slow. Note that, as in Theorem 3 in Cuevas et al. [13], Theorem 4.1 above does not require any continuity assumption on $F_{n}$.

Remark 4.2. Assumptions on gradient vector and Hessian matrix of $F$, in Theorems 3.1 and 4.1, are satisfied for a quite large class of classical bivariate distribution functions; for instance independent copula and exponential marginals, Farlie-Gumbel-Morgenstern (FGM), Clayton or Survival Clayton copulas and Burr marginals.

We now suppose that there exists a positive increasing sequences $\left(v_{n}\right)_{n \in \mathbb{N}^{*}}$ such that $v_{n}\left\|F-F_{n}\right\|_{\infty} \underset{n \rightarrow \infty}{\stackrel{\mathbb{P}}{\rightarrow}} 0$. Then

$$
\forall p \geq 1, \quad w_{n} \int_{\left[0, T_{n}\right]^{2}}\left|F-F_{n}\right|^{p} \lambda(\mathrm{d} x) \underset{n \rightarrow \infty}{\stackrel{\mathbb{P}}{\rightarrow}} 0, \quad \text { with } \quad w_{n}=\frac{v_{n}^{p}}{T_{n}^{2}} .
$$

In this case, from Theorem 4.1 we get

$$
p_{n}=o\left(w_{n}^{\frac{1}{p+1}} / T_{n}^{\frac{p}{p+1}}\right)=o\left(v_{n}^{\frac{p}{p+1}} / T_{n}^{\frac{p+2}{p+1}}\right) .
$$

From these considerations we can introduce the following remark.

Remark 4.3. Let $F_{n}$ the bivariate empirical distribution function. Then it holds that $v_{n}\left\|F-F_{n}\right\|_{\infty} \underset{n \rightarrow \infty}{\stackrel{\mathbb{P}}{\rightarrow}} 0$, with $v_{n}=o(\sqrt{n})$. From Theorem 4.1 and using the convergence rate in (4.1) with $p=2$, we obtain $p_{n}=$ $o\left(n^{\frac{1}{3}} / T_{n}^{\frac{4}{3}}\right)$. For instance, for $F(x, y)=\left(1-\mathrm{e}^{-x}\right)\left(1-\mathrm{e}^{-2 y}\right)$ and using the bivariate empirical distribution function $F_{n}$ we obtain $p_{n}=o\left(n^{\frac{1}{3}} / \ln (n)^{\frac{4}{3}}\right)$, with $p=2$ and $T_{n}=\ln (n)$.

\section{Application in Bivariate Risk theory}

From the usual definition in the univariate setting we know that the quantile function $Q_{X}$ provides a point which accumulates a probability $\alpha$ to the left tail and $1-\alpha$ to the right tail. More precisely, given an univariate continuous and strictly monotonic loss distribution function $F_{X}$,

$$
Q_{X}(\alpha)=F_{X}^{-1}(\alpha), \quad \forall \alpha \in(0,1) .
$$

The notion of univariate quantile function $Q_{X}$ is used in risk theory to define an univariate measure of risk: the Value-at-Risk (VaR). This measure is defined as

$$
\operatorname{VaR}_{\alpha}(X)=Q_{X}(\alpha), \quad \forall \alpha \in(0,1) .
$$


As presented in the introduction of this paper, following the general ideas of Embrechts and Puccetti [17] and Nappo and Spizzichino [24] an intuitive generalization of the VaR measure in the case of a bidimensional loss distribution function $F$ is given by its $\alpha$-quantile curves. More precisely:

Definition 5.1. For $\alpha \in(0,1)$ and $F \in \mathcal{F}$, the bidimensional Value-at-Risk at probability level $\alpha$ is the boundary of its $\alpha$-level set, i.e. $\operatorname{VaR}_{\alpha}(F)=\partial L(\alpha)$.

As pointed out in Tibiletti [32], imposing $\alpha=\frac{1}{2}$ in Definition 5.1, we get a natural extension of the bidimensional median. For details about a parametric formulation of the quantile curve $\partial L(\alpha)$ see Belzunce et al. [4]. For details about its properties see for instance Fernández-Ponce and Suárez-Lloréns [18] (and references therein) and Nappo and Spizzichino [24].

Then, using a bivariate estimator $F_{n}$ as in Sections 3 and 4 , we can define our plug-in estimator of the bivariate Value-at-Risk by

$$
\operatorname{VaR}_{\alpha}\left(F_{n}\right)=\partial L_{n}(\alpha), \quad \text { for } \alpha \in(0,1) .
$$

Moreover, under assumptions of Theorem 3.1, we obtain a consistency result, with respect to the Hausdorff distance, for the $\operatorname{VaR}_{\alpha}\left(F_{n}\right)$ on the quadrant $\mathbb{R}_{+}^{2}$ i.e.

$$
d_{H}\left(\operatorname{VaR}_{\alpha}(F)^{T_{n}}, \operatorname{VaR}_{\alpha}\left(F_{n}\right)^{T_{n}}\right)=O\left(\left\|F-F_{n}\right\|_{\infty}\right), \quad \text { a.s. }
$$

As in the univariate case, the two-dimensional $\mathrm{VaR}$ at a predetermined level $\alpha$ does not give any information about thickness of the upper tail of the distribution function. This is a considerable shortcoming of VaR measure because in practice we are not only concerned with frequency of the default but also with the severity of loss in case of default. In other words we are interested to analyze the behavior of $X$ and $Y$ not only on the boundary but also in the whole $\alpha$-level set.

In dimension one, in order to overcome this problem, another risk measure has recently received growing attention in insurance and finance literature: conditional tail expectation (CTE). Following Artzner et al. [1] and Dedu and Ciumara [15], for a continuous loss distribution function $F_{X}$ the CTE at level $\alpha$ is defined by

$$
\operatorname{CTE}_{\alpha}(X)=\mathbb{E}\left[X \mid X \geq \operatorname{VaR}_{\alpha}(X)\right]
$$

where $\operatorname{VaR}_{\alpha}(X)$ is the univariate $\operatorname{VaR}$ in (5.2). For a comprehensive treatment and for references to the extensive literature on $\operatorname{VaR}_{\alpha}(X)$ and $\mathrm{CTE}_{\alpha}(X)$ one may refer to Denuit et al. [16].

Several bivariate generalizations of the classical univariate CTE have been proposed; mainly using as conditioning events the total risk or some univariate aggregate extreme risk in the portfolio (for instance the sum, the max or the min between two risks):

$$
\begin{gathered}
\mathbb{E}\left[(X, Y) \mid X+Y>Q_{X+Y}(\alpha)\right], \quad \mathbb{E}\left[(X, Y) \mid \min \{X, Y\}>Q_{\min \{X, Y\}}(\alpha)\right] \text { and } \\
\mathbb{E}\left[(X, Y) \mid \max \{X, Y\}>Q_{\max \{X, Y\}}(\alpha)\right] .
\end{gathered}
$$

The interested reader is referred to Cai and $\mathrm{Li}[8]$ for further details. Using the same approach of Definition 5.1, we are interested to preserve the complete information about dependence structure between $X$ and $Y$ and the bi-dimensional aspect of our problem. To this end, starting from these general considerations, we propose to study a new bivariate version of the conditional tail expectation (Def. 5.2 below).

Let us first introduce the following assumption:

A2: $X$ and $Y$ are positive random variables fulfilling $\mathbb{E}\left(X^{2}\right)<\infty$ and $\mathbb{E}\left(Y^{2}\right)<\infty$. The random vector $(X, Y)$ has a $\lambda$-density function $f$ such that $\int f^{1+r} \mathrm{~d} \lambda<\infty$, for some $r>0$. 
Definition 5.2. Consider a random vector $(X, Y)$ satisfying Assumption A2, with associate distribution function $F \in \mathcal{F}$. For $\alpha \in(0,1)$, we define

(1) the bivariate $\alpha$-conditional tail expectation

$$
\operatorname{CTE}_{\alpha}(X, Y)=\mathbb{E}[(X, Y) \mid(X, Y) \in L(\alpha)]=\left(\begin{array}{l}
\mathbb{E}[X \mid(X, Y) \in L(\alpha)] \\
\mathbb{E}[Y \mid(X, Y) \in L(\alpha)]
\end{array}\right) .
$$

(2) the estimated bivariate $\alpha$-conditional tail expectation

$$
\widehat{\operatorname{CTE}}_{\alpha}(X, Y)=\left(\begin{array}{l}
\frac{\sum_{i=1}^{n} X_{i} 1_{\left\{\left(X_{i}, Y_{i}\right) \in L_{n}(\alpha)\right\}}}{\sum_{i=1}^{n} 1_{\left\{\left(X_{i}, Y_{i}\right) \in L_{n}(\alpha)\right\}}} \\
\frac{\sum_{i=1}^{n} Y_{i} 1_{\left\{\left(X_{i}, Y_{i}\right) \in L_{n}(\alpha)\right\}}}{\sum_{i=1}^{n} 1_{\left\{\left(X_{i}, Y_{i}\right) \in L_{n}(\alpha)\right\}}}
\end{array}\right) .
$$

Note that this bivariate conditional tail expectation is a natural extension of the univariate one. Moreover, if $X$ and $Y$ are identically distributed with a symmetric copula then $\mathbb{E}[X \mid(X, Y) \in L(\alpha)]=\mathbb{E}[Y \mid(X, Y) \in L(\alpha)]$.

Contrarily to the generalizations of the univariate $\mathrm{CTE}$ above, our $\mathrm{CTE}_{\alpha}(X, Y)$ does not use an aggregate variable (sum, min, $\max . .$.$) in order to analyze the bivariate risk's issue. By contrast, using a geometric$ approach, $\mathrm{CTE}_{\alpha}(X, Y)$ rather deals with the simultaneous joint damages considering the bivariate dependence structure of data in a specific risk's area $(L(\alpha))$.

Let $\alpha \in(0,1)$ and $F \in \mathcal{F}$. We introduce truncated versions of the theoretical and estimated $\mathrm{CTE}_{\alpha}$ :

$$
\begin{aligned}
& \operatorname{CTE}_{\alpha}^{T}(X, Y)=\mathbb{E}\left[(X, Y) \mid(X, Y) \in L(\alpha)^{T}\right], \\
& \widehat{\operatorname{CTE}}_{\alpha}^{T}(X, Y)=\left(\begin{array}{l}
\frac{\sum_{i=1}^{n} X_{i} 1_{\left\{\left(X_{i}, Y_{i}\right) \in L_{n}(\alpha)^{T}\right\}}}{\sum_{i=1}^{n} 1_{\left\{\left(X_{i}, Y_{i}\right) \in L_{n}(\alpha)^{T}\right\}}} \\
\frac{\sum_{i=1}^{n} Y_{i} 1_{\left\{\left(X_{i}, Y_{i}\right) \in L_{n}(\alpha)^{T}\right\}}}{\sum_{i=1}^{n} 1_{\left\{\left(X_{i}, Y_{i}\right) \in L_{n}(\alpha)^{T}\right\}}}
\end{array}\right),
\end{aligned}
$$

where $L(\alpha)^{T}$ and $L_{n}(\alpha)^{T}$ are the truncated versions of theoretical and estimated upper $\alpha$-level set defined in Section 2.

Theorem 5.3. Under Assumption A2, Assumptions of Theorem 4.1 and with the notation of Theorem 4.1, it holds that

$$
\beta_{n}\left|\operatorname{CTE}_{\alpha}^{T_{n}}(X, Y)-{\widehat{\mathrm{CTE}_{\alpha}}}_{\alpha}^{T_{n}}(X, Y)\right| \underset{n \rightarrow \infty}{\stackrel{\mathbb{P}}{\rightarrow}} 0
$$

where $\beta_{n}=\min \left\{p_{n}^{\frac{r}{2(1+r)}}, a_{n}\right\}$, with $r>0$ satisfying Assumption $\mathbf{A 2}$ and $a_{n}=o(\sqrt{n})$.

The convergence in (5.4) must be interpreted as a componentwise convergence. In the case of a bounded density function $f_{(X, Y)}$ we obtain $\beta_{n}=\min \left\{\sqrt{p_{n}}, a_{n}\right\}$.

Using Theorem 5.3 and Remark 4.3, we obtain the following result in the case of the bivariate empirical distribution function.

Corollary 5.4. Let $F_{n}$ the bivariate empirical distribution function. Under Assumptions Theorem 5.3 and with the notation of Theorem 5.3, it holds that

$$
\beta_{n}\left|\operatorname{CTE}_{\alpha}^{T_{n}}(X, Y)-\widehat{\operatorname{CTE}}_{\alpha}^{T_{n}}(X, Y)\right| \underset{n \rightarrow \infty}{\stackrel{\mathbb{P}}{\rightarrow}} 0,
$$

where $\beta_{n}=o\left(n^{\frac{r}{6(1+r)}} / T_{n}^{\frac{2 r}{3(1+r)}}\right)$, with $r>0$ satisfying Assumption A2.

In the case of a bounded density function $f_{(X, Y)}, \beta_{n}=o\left(n^{\frac{1}{6}} / T_{n}^{\frac{2}{3}}\right)$.

Remark 5.5. Starting from Corollary 5.4, it could be interesting to consider the convergence $\left|\mathrm{CTE}_{\alpha}(X, Y)-\widehat{\mathrm{CTE}}_{\alpha}^{T}(X, Y)\right|$. We remark that in this case the speed of convergence will also depend on the 
convergence rate to zero of $\left|\operatorname{CTE}_{\alpha}(X, Y)-\operatorname{CTE}_{\alpha}^{T_{n}}(X, Y)\right|$, then, in particular of $\mathbb{P}\left[(X, Y) \in L(\alpha) \backslash L(\alpha)^{T_{n}}\right]$ for $n \rightarrow \infty$. More precisely $\left|\mathrm{CTE}_{\alpha}(X, Y)-\mathrm{CTE}_{\alpha}^{T_{n}}(X, Y)\right|$ decays to zero at least with a convergence rate $\left(\mathbb{P}\left[X \geq T_{n} \text { or } Y \geq T_{n}\right]\right)^{-1}$. We now provide an illustration (in a simple case) on how to choose $T_{n}$ in order to consider the convergence $\left|\mathrm{CTE}_{\alpha}(X, Y)-\widehat{\mathrm{CTE}}_{\alpha}^{T_{n}}(X, Y)\right|$. Let $F(x, y)=\left(1-\mathrm{e}^{-x}\right)\left(1-\mathrm{e}^{-y}\right)$, i.e. independent and exponentially distributed marginals with parameter 1 , with bounded density function in $\mathbb{R}_{+}^{2}$. Let $F_{n}$ the bivariate empirical distribution function. In this case all assumptions of Corollary 5.4 are satisfied. Then we obtain that $\left|\mathrm{CTE}_{\alpha}(X, Y)-\widehat{\mathrm{CTE}}_{\alpha}^{T_{n}}(X, Y)\right|$ decays to zero at least with a convergence rate $\beta_{n}=o\left(n^{\frac{1}{6}} / \ln (n)^{\frac{2}{3}}\right)$, with a choice of sequence $T_{n}=\ln (n)$. We remark that $\left(\mathbb{P}\left[X \geq T_{n} \text { or } Y \geq T_{n}\right]\right)^{-1}$ is increasing in $T_{n}$, whereas the speed of convergence is decreasing in $T_{n}$. This kind of compromise provides an illustration on how to choose $T_{n}$, apart from satisfying the assumptions of consistency results in Section 4 (see Sect. 6.2.1 for several numerical illustrations of this aspect).

\section{ILLUSTRATiOnS}

\subsection{Estimation of the level sets}

In this section we confront our estimator of level sets of the distribution function with various simulated samples. The plug-in estimation of level sets is constructed using the empirical estimator $F_{n}$. We consider two distribution functions which satisfy assumptions of Theorem 4.1: independent copula with exponential marginals and Survival Clayton copula with Burr marginals.

Following Remark 4.3 we consider $T_{n}=\ln (n)$ and we obtain $p_{n}=o\left(n^{\frac{1}{3}} / \ln (n)^{\frac{4}{3}}\right)$. We also take $T_{n}=n^{0.45}$. This second sequence provides an illustration for the case of $T_{n}$ too large (see Tabs. $1 \mathrm{~b}$ and $2 \mathrm{~b}, 4 \mathrm{~b}$ and $5 \mathrm{~b}$ ).

We take a random grid of 10000 points in $\left[0, T_{n}\right]^{2}$ and a distribution with independent and exponentially distributed marginals with parameter 1 and 2 respectively. We provide a Monte Carlo approximation for $\lambda\left(L(\alpha)^{T_{n}} \triangle L_{n}(\alpha)^{T_{n}}\right)$ (averaged on 100 iterations), for different values of $\alpha$ and $n$. The results are gathered in Table 1.

In order to illustrate the influence of the choice of $T_{n}$, in Table 2 we show $p_{n} \lambda\left(L(\alpha)^{T_{n}} \triangle L_{n}(\alpha)^{T_{n}}\right)$, with $p_{n}=o\left(n^{\frac{1}{3}} / \ln (n)^{\frac{4}{3}}\right)$ in the case $T_{n}=\ln (n)$ (Tab. 2a) and $T_{n}=n^{0.45}$ (Tab. 2b). Results in Table 2a set out how $p_{n}=o\left(n^{\frac{1}{3}} / \ln (n)^{\frac{4}{3}}\right)$ is at least the convergence rate of $\lambda\left(L(\alpha)^{T_{n}} \triangle L_{n}(\alpha)^{T_{n}}\right)$ in this particular case. This illustrates not only the consistency property but also the speed of convergence of our plug-in estimator. As expected in Table $2 \mathrm{~b}$ we see that taking $T_{n}$ too large, we can not expect to get a good approximation of $\lambda\left(L(\alpha)^{T_{n}} \triangle L_{n}(\alpha)^{T_{n}}\right)$.

In Tables 3a and 3b we calculate the theoretical $\lambda\left(L(\alpha)^{T_{n}}\right)$, for $T_{n}=\ln (n)$ and $T_{n}=n^{0.45}$ respectively, and for different values of $n$ and $\alpha$. These quantities provide an illustration of the truncation of level sets $L(\alpha)$, with respect to $T_{n}$ and $\alpha$.

Table 4 provides a Monte Carlo approximation for $\lambda\left(L(\alpha)^{T_{n}} \triangle L_{n}(\alpha)^{T_{n}}\right)$ (averaged on 100 iterations), in the case of a Survival Clayton copula with parameter 1 and Burr $(2,1)$ marginals. In Table 5 we show $p_{n} \lambda\left(L(\alpha)^{T_{n}} \triangle\right.$ $L_{n}(\alpha)^{T_{n}}$ ), with $p_{n}=o\left(n^{\frac{1}{3}} / \ln (n)^{\frac{4}{3}}\right)$ in the case $T_{n}=\ln (n)$ (Tab. 5a) and $T_{n}=n^{0.45}$ (Tab. 5b). We obtain results that are analogous to those Table 2 .

As expected, the greater $n$ is, the better the estimations are (see Tabs. 1a and 4b). Moreover we note that for big values of $\alpha$ we need more data to get a good estimation of the level sets. This may come from the fact that when $\alpha$ grows the gradient of the distribution function decreases to zero and the constant $A$ grows significantly (see proof of Theorem 4.1 in Sect. 8).

Remark 6.1. As remarked in Section 4 the choice of the sequence $T_{n}$ is essentially a compromise: if $T_{n}$ is large we have a quite slow convergence rate $p_{n}$ for $\lambda\left(L(\alpha)^{T_{n}} \triangle L_{n}(\alpha)^{T_{n}}\right)$ and if $T_{n}$ is quite small we obtain a faster convergence rate. However choosing $T_{n}$ too small we substantially truncate our level sets. This aspect can be problematic in the case $c$ is close to one. Finally the theoretical optimal criterion for the choice of sequence $T_{n}$ in Theorem 4.1 is an open interesting problem that we leave for a future work. 
TABLE 1. Distribution with independent and exponentially distributed marginals with parameter 1 and 2 respectively. Approximated $\lambda\left(L(\alpha)^{T_{n}} \triangle L_{n}(\alpha)^{T_{n}}\right)$.

\begin{tabular}{|c|c|c|c|}
\hline$\alpha$ & $n=500$ & $n=1000$ & $n=2000$ \\
\hline 0.10 & 0.099 & 0.089 & 0.078 \\
0.24 & 0.226 & 0.176 & 0.075 \\
0.38 & 0.248 & 0.183 & 0.143 \\
0.52 & 0.324 & 0.223 & 0.217 \\
0.66 & 0.429 & 0.259 & 0.232 \\
0.80 & 0.613 & 0.371 & 0.332 \\
\hline
\end{tabular}

(b) $T_{n}=\ln (n)$

\begin{tabular}{|c|c|c|c|}
\hline$\alpha$ & $n=500$ & $n=1000$ & $n=2000$ \\
\hline 0.10 & 0.331 & 0.326 & 0.223 \\
0.24 & 0.519 & 0.391 & 0.249 \\
0.38 & 0.591 & 0.469 & 0.396 \\
0.52 & 1.057 & 0.906 & 0.881 \\
0.66 & 1.222 & 0.989 & 0.904 \\
0.80 & 1.541 & 1.367 & 1.334 \\
\hline
\end{tabular}

(b) $T_{n}=n^{0.45}$

TABLE 2. Distribution with independent and exponentially distributed marginals with parameter 1 and 2 respectively. Approximated $p_{n} \lambda\left(L(\alpha)^{T_{n}} \triangle L_{n}(\alpha)^{T_{n}}\right)$, with $p_{n}=o\left(n^{\frac{1}{3}} / \ln (n)^{\frac{4}{3}}\right)$.

\begin{tabular}{|c|c|c|c|}
\hline$\alpha$ & $n=500$ & $n=1000$ & $n=2000$ \\
\hline 0.10 & 0.069 & 0.068 & 0.065 \\
0.24 & 0.156 & 0.134 & 0.063 \\
0.38 & 0.172 & 0.139 & 0.121 \\
0.52 & 0.225 & 0.169 & 0.183 \\
0.66 & 0.298 & 0.199 & 0.195 \\
0.80 & 0.426 & 0.282 & 0.279 \\
\hline
\end{tabular}

(a) $T_{n}=\ln (n)$

\begin{tabular}{|c|c|c|c|}
\hline$\alpha$ & $n=500$ & $n=1000$ & $n=2000$ \\
\hline 0.10 & 0.229 & 0.248 & 0.188 \\
0.24 & 0.361 & 0.298 & 0.209 \\
0.38 & 0.411 & 0.357 & 0.339 \\
0.52 & 0.734 & 0.689 & 0.743 \\
0.66 & 0.849 & 0.752 & 0.762 \\
0.80 & 1.071 & 1.039 & 1.124 \\
\hline
\end{tabular}

(b) $T_{n}=n^{0.45}$

TABLE 3. Theoretical $\lambda\left(L(\alpha)^{T_{n}}\right)$, for different values of $n$ and $\alpha$. Distribution with independent and exponentially distributed marginals with parameter 1 and 2 respectively.

\begin{tabular}{|c|c|c|c|}
\hline$\alpha$ & $n=500$ & $n=1000$ & $n=2000$ \\
\hline 0.10 & 37.472 & 46.459 & 56.405 \\
0.24 & 35.777 & 44.588 & 54.358 \\
0.38 & 33.837 & 42.436 & 51.995 \\
0.52 & 31.505 & 39.837 & 49.129 \\
0.66 & 28.513 & 36.487 & 45.421 \\
0.80 & 24.201 & 31.621 & 40.003 \\
\hline
\end{tabular}

(a) $T_{n}=\ln (n)$

\begin{tabular}{|c|c|c|c|}
\hline$\alpha$ & $n=500$ & $n=1000$ & $n=2000$ \\
\hline 0.10 & 142.205 & 248.516 & 433.872 \\
0.24 & 139.039 & 244.378 & 428.449 \\
0.38 & 135.328 & 239.495 & 422.021 \\
0.52 & 130.771 & 233.464 & 414.045 \\
0.66 & 124.779 & 225.488 & 403.451 \\
0.80 & 115.851 & 213.505 & 387.436 \\
\hline
\end{tabular}

(b) $T_{n}=n^{0.45}$

TABLE 4. Distribution with Survival Clayton copula with parameter 1 and $\operatorname{Burr}(2,1)$ marginals. Approximated $\lambda\left(L(\alpha)^{T_{n}} \triangle L_{n}(\alpha)^{T_{n}}\right)$.

\begin{tabular}{|c|c|c|c|}
\hline$\alpha$ & $n=500$ & $n=1000$ & $n=2000$ \\
\hline 0.10 & 0.208 & 0.171 & 0.126 \\
0.24 & 0.252 & 0.241 & 0.149 \\
0.38 & 0.339 & 0.288 & 0.132 \\
0.52 & 0.349 & 0.335 & 0.245 \\
0.66 & 0.499 & 0.364 & 0.353 \\
0.80 & 0.771 & 0.644 & 0.605 \\
\hline
\end{tabular}

(b) $T_{n}=\ln (n)$

\begin{tabular}{|c|c|c|c|}
\hline$\alpha$ & $n=500$ & $n=1000$ & $n=2000$ \\
\hline 0.10 & 0.697 & 0.633 & 0.536 \\
0.24 & 0.893 & 0.872 & 0.809 \\
0.38 & 0.971 & 0.911 & 0.879 \\
0.52 & 1.001 & 0.982 & 1.229 \\
0.66 & 1.569 & 1.522 & 1.413 \\
0.80 & 2.377 & 2.269 & 2.175 \\
\hline
\end{tabular}

(b) $T_{n}=n^{0.45}$ 
TABle 5. Distribution with Survival Clayton copula with parameter 1 and Burr $(2,1)$ marginals. Approximated $p_{n} \lambda\left(L(\alpha)^{T_{n}} \triangle L_{n}(\alpha)^{T_{n}}\right)$, with $p_{n}=o\left(n^{\frac{1}{3}} / \ln (n)^{\frac{4}{3}}\right)$.

\begin{tabular}{|c|c|c|c|}
\hline$\alpha$ & $n=500$ & $n=1000$ & $n=2000$ \\
\hline 0.10 & 0.144 & 0.129 & 0.106 \\
0.24 & 0.175 & 0.183 & 0.126 \\
0.38 & 0.235 & 0.219 & 0.111 \\
0.52 & 0.242 & 0.254 & 0.206 \\
0.66 & 0.347 & 0.277 & 0.297 \\
0.80 & 0.535 & 0.489 & 0.501 \\
\hline
\end{tabular}

(b) $T_{n}=\ln (n)$

\begin{tabular}{|c|c|c|c|}
\hline$\alpha$ & $n=500$ & $n=1000$ & $n=2000$ \\
\hline 0.10 & 0.484 & 0.481 & 0.452 \\
0.24 & 0.620 & 0.663 & 0.682 \\
0.38 & 0.674 & 0.692 & 0.741 \\
0.52 & 0.695 & 0.746 & 1.036 \\
0.66 & 1.089 & 1.157 & 1.191 \\
0.80 & 1.651 & 1.725 & 1.834 \\
\hline
\end{tabular}

(b) $T_{n}=n^{0.45}$

\subsection{Estimation of $\mathrm{CTE}_{\alpha}(\boldsymbol{X}, \boldsymbol{Y})$}

\subsubsection{Simulated data}

In order to evaluate the performance of our estimator we present here some simulated cases of estimation of $\mathrm{CTE}_{\alpha}(X, Y)$, for different values of level $\alpha$. To compare the estimated results with the theoretical ones we consider cases for which we can calculate (with Maple) the explicit value of the theoretical $\mathrm{CTE}_{\alpha}(X, Y)$. However our estimator can be applied to much more general cases. By being able to get a good estimator for the level sets, we can expect a good estimator for $\operatorname{CTE}_{\alpha}(X, Y)$.

In the following we compare $\widehat{\mathrm{CTE}}_{\alpha}^{T_{n}}(X, Y)$ with the theoretical $\mathrm{CTE}_{\alpha}(X, Y)$, for different distributions: Independent copula with exponentially distributed marginals; Clayton copula with parameter 1 , with exponential and Burr $(4,1)$ univariate marginals. The plug-in estimation of level sets is constructed using the empirical estimator $F_{n}$ of the bivariate distribution function, with $n=1000$.

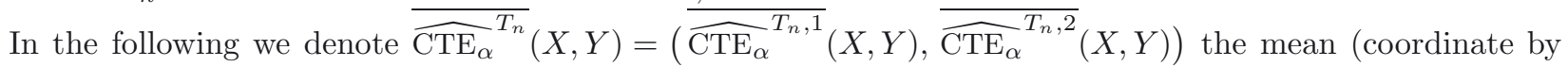
coordinate) of $\widehat{\mathrm{CTE}}_{\alpha}^{T_{n}}(X, Y)$ on 100 simulations. We denote $\widehat{\sigma}=\left(\widehat{\sigma}_{1}, \widehat{\sigma}_{2}\right)$ the empirical standard deviation (coordinate by coordinate) with

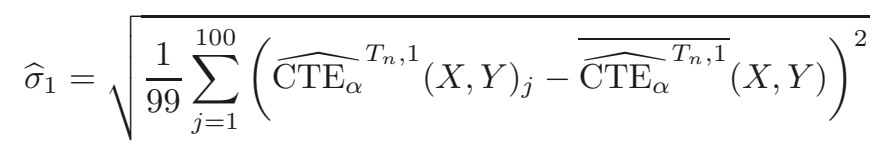

relatives to the first coordinate (resp. $\widehat{\sigma}_{2}$ relatives to the second one).

We denote $\mathrm{RMSE}=\left(\mathrm{RMSE}_{1}, \mathrm{RMSE}_{2}\right)$ the relative mean square error (coordinate by coordinate) with

$$
\operatorname{RMSE}_{1}=\sqrt{\frac{1}{100} \sum_{j=1}^{100}\left(\frac{\widehat{\mathrm{CTE}}_{\alpha}^{T_{n}, 1}(X, Y)_{j}-\mathrm{CTE}_{\alpha}^{T_{n}, 1}(X, Y)}{\mathrm{CTE}_{\alpha}^{T_{n}, 1}(X, Y)}\right)^{2}}
$$

relatives to the first coordinate of $\mathrm{CTE}_{\alpha}^{T_{n}}(X, Y)$ (resp. $\mathrm{RMSE}_{2}$ relatives to the second one).

For the Independent copula with exponentially distributed marginals, from Corollary 5.4 and Remark 5.5, we take $T_{n}=\ln (n)$ (see Tabs. 6 and 8$)$. This choice provides a convergence rate $\beta_{n}=o\left(n^{\frac{1}{6}} / \ln (n)^{\frac{2}{3}}\right)$. Furthermore, as in Section 6.1, we consider the case with $T_{n}=n^{0.45}$. This second sequence provides an illustration for the case of $T_{n}$ too large (see Tabs. 7 and 9). Following the same approach of Remark 5.5, in the case of Clayton copula with parameter 1, with exponential and $\operatorname{Burr}(4,1)$ univariate marginals we have $\beta_{n}=o\left(n^{\frac{1}{6}} /\left(n^{0.2}\right)^{\frac{2}{3}}\right)$, with a sequence $T_{n}=n^{0.2}$ (see Tab. 10). Also in this case, we show the behavior of our estimator for $T_{n}=n^{0.45}$ (see Tab. 11). 
TABLE 6. $(X, Y)$ with independent and exponentially distributed components with parameter 2; $T_{n}=\ln (n)$.

\begin{tabular}{|c|c|c|c|c|}
\hline$\alpha$ & $\mathrm{CTE}_{\alpha}(X, Y)$ & $\overline{\widehat{\mathrm{CTE}}_{\alpha}^{T_{n}}}(X, Y)$ & $\widehat{\sigma}$ & $\mathrm{RMSE}$ \\
\hline 0.10 & $(0.627,0.627)$ & $(0.585,0.637)$ & $(0.022,0.021)$ & $(0.035,0.039)$ \\
0.24 & $(0.761,0.761)$ & $(0.766,0.759)$ & $(0.031,0.031)$ & $(0.041,0.046)$ \\
0.38 & $(0.896,0.896)$ & $(0.864,0.917)$ & $(0.036,0.037)$ & $(0.053,0.049)$ \\
0.52 & $(1.051,1.051)$ & $(1.027,1.071)$ & $(0.051,0.053)$ & $(0.055,0.052)$ \\
0.66 & $(1.246,1.246)$ & $(1.252,1.238)$ & $(0.075,0.067)$ & $(0.063,0.065)$ \\
0.80 & $(1.531,1.531)$ & $(1.497,1.549)$ & $(0.123,0.117)$ & $(0.083,0.077)$ \\
\hline
\end{tabular}

TABLE 7. $(X, Y)$ with independent and exponentially distributed components with parameter 2; $T_{n}=n^{0.45}$.

\begin{tabular}{|c|c|c|c|c|}
\hline$\alpha$ & $\operatorname{CTE}_{\alpha}(X, Y)$ & ${\overline{\widehat{\mathrm{CTE}}_{\alpha}}}^{T_{n}}(X, Y)$ & $\widehat{\sigma}$ & RMSE \\
\hline 0.10 & $(0.627,0.627)$ & $(0.603,0.656)$ & $(0.031,0.031)$ & $(0.062,0.068)$ \\
\hline 0.24 & $(0.761,0.761)$ & $.774,0.731)$ & $(0.061,0$ & $(0.082,0.130)$ \\
\hline 0.38 & $(0.896,0.896)$ & $(0.927,0.871)$ & $(0.072,0.076)$ & $(0.087,0.119)$ \\
\hline 0.52 & $(1.051,1.051)$ & $1.086,1.128)$ & $(0.094,0.082)$ & $(0.095,0.107)$ \\
\hline 0.66 & $(1.246,1.246)$ & $(1.281,1.322)$ & $(0.127,0.101)$ & $(0.102,0.101)$ \\
\hline 0.80 & $(1.531,1.531)$ & $(1.545,1.611)$ & $(0.157,0.161)$ & $(0.105,0.117)$ \\
\hline
\end{tabular}

TABLE 8. $(X, Y)$ with independent and exponentially distributed components with parameter 1 and 2 respectively; $T_{n}=\ln (n)$.

\begin{tabular}{|c|c|c|c|c|}
\hline$\alpha$ & $\operatorname{CTE}_{\alpha}(X, Y)$ & ${\overline{\widehat{\mathrm{CTE}}_{\alpha}}}^{T_{n}}(X, Y)$ & $\widehat{\sigma}$ & RMSE \\
\hline 0.10 & $(1.255,0.627)$ & $(1.222,0.638)$ & $(0.044,0.022)$ & $(0.043,0.039)$ \\
\hline 0.24 & $(1.521,0.761)$ & $(1.488,0.811)$ & $(0.069,0.023)$ & $(0.051,0.042)$ \\
\hline 0.38 & $(1.792,0.896)$ & $(1.797,0.911)$ & $(0.075,0.038)$ & $(0.044,0.046)$ \\
\hline 0.52 & $(2.102,1.051)$ & $(2.082,1.047)$ & $(0.104,0.052)$ & $(0.052,0.052)$ \\
\hline 0.66 & $(2.492,1.246)$ & $(2.461,1.255)$ & $(0.139,0.071)$ & $(0.057,0.056)$ \\
\hline 0.80 & $(3.061,1.531)$ & $(3.011,1.544)$ & $(0.251,0.125)$ & $(0.084,0.082)$ \\
\hline
\end{tabular}

TABLE 9. $(X, Y)$ with independent and exponentially distributed components with parameter 1 and 2 respectively; $T_{n}=n^{0.45}$.

\begin{tabular}{|c|c|c|c|c|}
\hline$\alpha$ & $\mathrm{CTE}_{\alpha}(X, Y)$ & ${\overline{\widehat{\mathrm{CTE}}_{\alpha}}}^{T_{n}}(X, Y)$ & $\widehat{\sigma}$ & RMSE \\
\hline 0.10 & $(1.255,0.627)$ & $(1.253,0.624)$ & $(0.061,0.043)$ & $(0.051,0.054)$ \\
\hline 0.24 & $(1.521,0.761)$ & $(1.514,0.803)$ & $(0.074,0.049)$ & $(0.048,0.075)$ \\
\hline 0.38 & $(1.792,0.896)$ & $(1.793,0.948)$ & $(0.096,0.055)$ & $(0.053,0.084)$ \\
\hline 0.52 & $(2.102,1.051)$ & $(2.087,1.111)$ & $(0.118,0.076)$ & $(0.056,0.092)$ \\
\hline 0.66 & $(2.492,1.246)$ & $(2.497,1.311)$ & $(0.169,0.108)$ & $(0.068,0.101)$ \\
\hline 0.80 & $(3.061,1.531)$ & $(3.056,1.602)$ & $(0.313,0.153)$ & $(0.102,0.111)$ \\
\hline
\end{tabular}


TABle 10. $(X, Y)$ with Clayton copula with parameter $1, F_{X}$ exponential distribution with parameter $1, F_{Y} \operatorname{Burr}(4,1)$ distribution; $T_{n}=n^{0.2}$.

\begin{tabular}{|c|c|c|c|c|}
\hline$\alpha$ & $\mathrm{CTE}_{\alpha}(X, Y)$ & $\overline{{\widehat{\mathrm{CTE}_{\alpha}}}^{T_{n}}}(X, Y)$ & $\widehat{\sigma}$ & $\operatorname{RMSE}$ \\
\hline 0.10 & $(1.188,1.229)$ & $(1.049,1.192)$ & $(0.032,0.021)$ & $(0.019,0.033)$ \\
0.24 & $(1.448,1.366)$ & $(1.283,1.379)$ & $(0.053,0.224)$ & $(0.019,0.063)$ \\
0.38 & $(1.727,1.505)$ & $(1.525,1.471)$ & $(0.046,0.031)$ & $(0.019,0.031)$ \\
0.52 & $(2.049,1.666)$ & $(1.803,1.625)$ & $(0.058,0.041)$ & $(0.023,0.034)$ \\
0.66 & $(2.454,1.875)$ & $(2.129,1.823)$ & $(0.071,0.054)$ & $(0.035,0.039)$ \\
0.80 & $(3.039,2.202)$ & $(2.591,2.144)$ & $(0.111,0.105)$ & $(0.055,0.054)$ \\
\hline
\end{tabular}

TABle 11. $(X, Y)$ with Clayton copula with parameter $1, F_{X}$ exponential distribution with parameter 1, $F_{Y} \operatorname{Burr}(4,1)$ distribution; $T_{n}=n^{0.45}$.

\begin{tabular}{|c|c|c|c|c|}
\hline$\alpha$ & $\operatorname{CTE}_{\alpha}(X, Y)$ & $\overline{{\widehat{\mathrm{CTE}_{\alpha}}}^{T_{n}}}(X, Y)$ & $\widehat{\sigma}$ & RMSE \\
\hline 0.10 & $(1.188,1.229)$ & $(1.189,1.238)$ & $(0.061,0.035)$ & $(0.039,0.029)$ \\
\hline 0.24 & $(1.448,1.366)$ & $(1.462,1.365)$ & $(0.065,0.037)$ & $(0.046,0.031)$ \\
\hline 0.38 & $(1.727,1.505)$ & $(1.751,1.536)$ & $(0.082,0.046)$ & $(0.049,0.037)$ \\
\hline 0.52 & $(2.049,1.666)$ & $(2.063,1.713)$ & $(0.091,0.061)$ & $(0.051,0.045)$ \\
\hline 0.66 & $(2.454,1.875)$ & $(2.457,1.951)$ & $(0.117,0.104)$ & $(0.057,0.068)$ \\
\hline 0.80 & $(3.039,2.202)$ & $(3.037,2.322)$ & $(0.192,0.165)$ & $(0.063,0.108)$ \\
\hline
\end{tabular}

TABLE 12. Evolution of $\widehat{\sigma}$ and RMSE in terms of sample size $n$ for $\alpha=0.9 ;(X, Y)$ independent and exponentially distributed components with parameter 1 and 2 respectively. $T_{n}=\ln (n)$.

\begin{tabular}{|c|c|c|c|c|c|c|}
\hline$n$ & 500 & 1000 & 1500 & 2000 & 2500 & 5000 \\
\hline$\widehat{\sigma}$ & $(0.614,0.359)$ & $(0.444,0.308)$ & $(0.431,0.295)$ & $(0.377,0.168)$ & $(0.241,0.123)$ & $(0.216,0.121)$ \\
\hline $\operatorname{RMSE}$ & $(0.168,0.189)$ & $(0.123,0.163)$ & $(0.115,0.161)$ & $(0.099,0.089)$ & $(0.077,0.079)$ & $(0.063,0.057)$ \\
\hline
\end{tabular}

TABLE 13. Evolution of $\widehat{\sigma}$ and RMSE in terms of sample size $n$ for $\alpha=0.9 ;(X, Y)$ independent and exponentially distributed components with parameter 1 and 2 respectively. $T_{n}=n^{0.45}$.

\begin{tabular}{|c|c|c|c|c|c|c|}
\hline$n$ & 500 & 1000 & 1500 & 2000 & 2500 & 5000 \\
\hline$\widehat{\sigma}$ & $(0.919,0.419)$ & $(0.568,0.319)$ & $(0.511,0.294)$ & $(0.382,0.239)$ & $(0.348,0.223)$ & $(0.307,0.151)$ \\
\hline RMSE & $(0.242,0.221)$ & $(0.151,0.172)$ & $(0.133,0.165)$ & $(0.101,0.144)$ & $(0.093,0.129)$ & $(0.092,0.108)$ \\
\hline
\end{tabular}

Following we show that for high levels (here $\alpha=0.9$ ), one needs to use large samples to get reasonable estimates of $\mathrm{CTE}_{\alpha}$. We consider for the purpose $(X, Y)$ independent and exponentially distributed with respective parameters 1 and 2. The theoretical value is $\mathrm{CTE}_{0.9}(X, Y)=(3.78,1.89)$. We take $T_{n}=\ln (n)$ (see Tab. 12). In this case we need between 2000 and 2500 data to get the same performances as for lower level (see Tab. 8 above). Furthermore we consider the case $T_{n}=n^{0.45}$ (see Tab. 13). In this case we need between 2500 and 5000 data to get the same performances as for lower level (see Tab. 9 above). 
The bad properties of the estimate for sample sizes less than 2000 (in the case of $T_{n}=\ln (n)$ ) can be explained by the fact that for high levels, the constant $A$ is large (see Proof of Theorem 4.1 in Sect. 8), but also by the fact that for large values of $\alpha$ the empirical estimate $F_{n}$ of $F$ may not be the most appropriate.

\subsubsection{Real data}

We consider here the estimation of $\mathrm{CTE}_{\alpha}$ in a real case: Loss-ALAE data in the log scale (for details see Frees and Valdez [19]). Each claim consists of an indemnity payment (the loss, $X$ ) and an allocated loss adjustment expense (ALAE, $Y$ ). Examples of ALAE are the fees paid to outside attorneys, experts, and investigators used to defend claims.

The data size is $n=1500$. Let $T_{n}=n^{0.4}$. Again our estimator is constructed using the empirical estimator $F_{n}$. Results about the estimation of $\mathrm{CTE}_{\alpha}^{T_{n}}$ are gathered in Table 14. In Figure 2 we represent data, estimated level sets and estimated bivariate conditional tail expectation for several values of $\alpha$.

In this real setting the estimation of $\mathrm{CTE}_{\alpha}$ can be used in order to quantify the mean of the Loss (resp. ALAE) in the log scale conditionally to the fact that Loss and ALAE data belong jointly to the specific risk's area $L(\alpha)$.
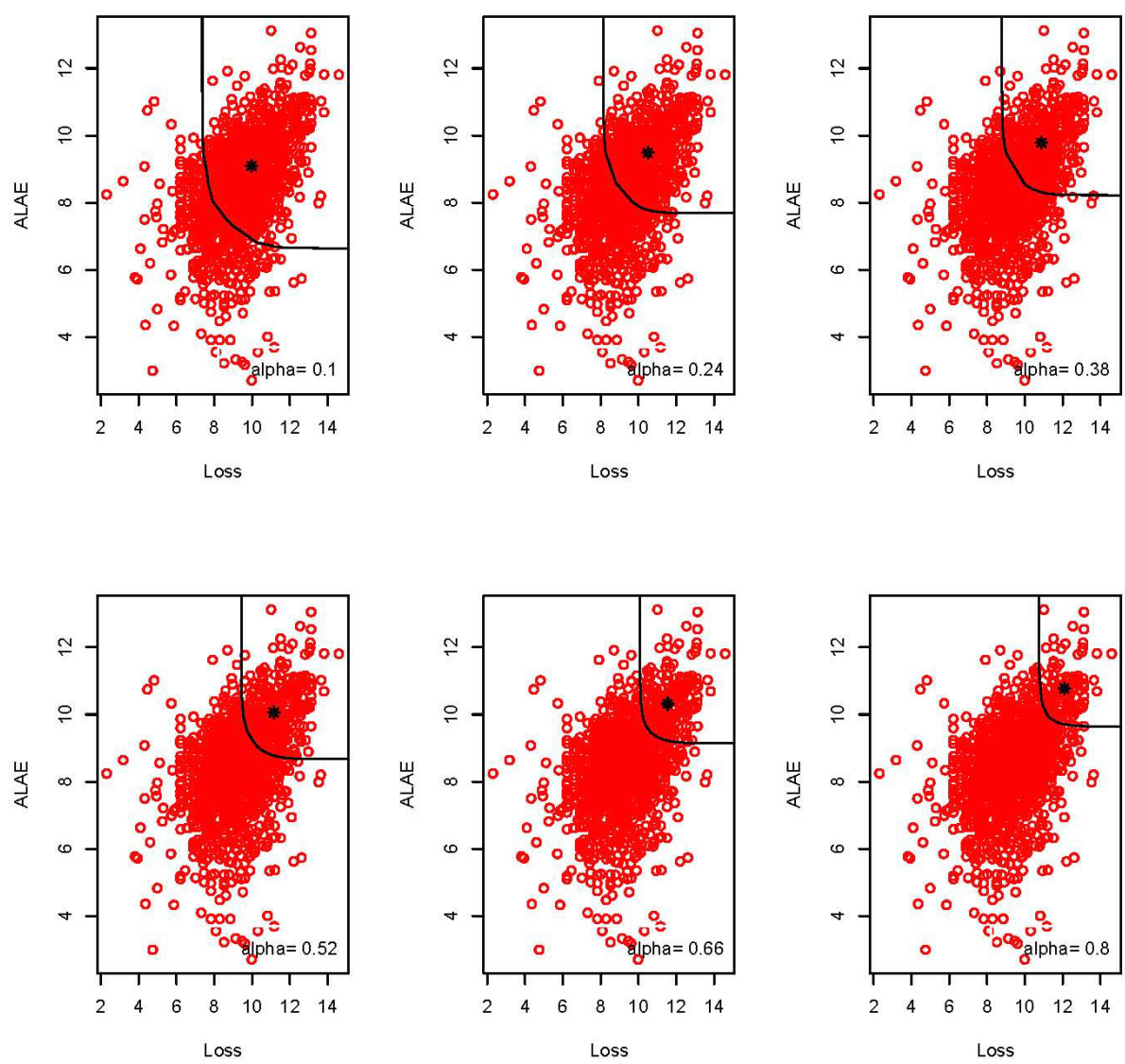

Figure 2. Loss-ALAE data in log scale: boundary of estimated level sets (line) and $\widehat{\mathrm{CTE}}_{\alpha}^{T_{n}}$ (star) for different values of $\alpha$. 
TABLE 14. $\widehat{\mathrm{CTE}}_{\alpha}^{T_{n}}$ for Loss-ALAE data in log scale, with different values of level $\alpha$.

\begin{tabular}{|c|c|c|c|}
\hline$\alpha$ & 0.10 & 0.24 & 0.38 \\
\hline$\widehat{\mathrm{CTE}}_{\alpha}^{T_{n}}$ & $(9.937,9.252)$ & $(10.361,9.566)$ & $(10.731,9.728)$ \\
\hline \hline$\alpha$ & 0.52 & 0.66 & 0.80 \\
\hline$\widehat{\mathrm{CTE}}_{\alpha}^{T_{n}}$ & $(11.096,10.011)$ & $(11.518,10.315)$ & $(12.057,10.758)$ \\
\hline
\end{tabular}

\section{Conclusion}

In this paper we have provided convergence results for the plug-in estimator of the level sets of an unknown distribution function on $\mathbb{R}_{+}^{2}$ in terms of Hausdorff distance and volume of the symmetric difference. In this setting we have proposed and estimated a new bivariate risk measure: $\mathrm{CTE}_{\alpha}(X, Y)$. A future work comparing our bivariate Conditional Tail Expectation with existing bivariate generalizations of the CTE in terms of classical properties (monotonicity, translation invariance, homogeneity, ...), dependence structure, behavior with respect to different risk scenarios, is in preparation.

\section{PROOFS}

Proof of Proposition 2.1. Take $T>0$ such that for all $t:|t-c| \leq r,\{F=t\}^{T} \neq \emptyset$, (from assumptions of Proposition 2.1 we know that such an $T$ there exists).

Let $x \in\left\{z \in[0, T]^{2}:|F(z)-c| \leq r\right\}$. Define for $\bar{\lambda} \in \mathbb{R}$

$$
y_{\bar{\lambda}} \equiv y_{\bar{\lambda}, x}=x+\bar{\lambda} \frac{(\nabla F)_{x}}{\left\|(\nabla F)_{x}\right\|},
$$

so $\left\|y_{\bar{\lambda}}-x\right\|=|\bar{\lambda}|$. From the differentiability properties of $F$ and using Taylor's formula we have, for $|\bar{\lambda}|<\zeta$

$$
F\left(y_{\bar{\lambda}}\right)=F(x)+(\nabla F)_{x}^{T}\left(y_{\bar{\lambda}}-x\right)+\frac{1}{2}\left(y_{\bar{\lambda}}-x\right)^{T}(H F)_{\bar{x}}\left(y_{\bar{\lambda}}-x\right),
$$

with $\bar{x}$ a point on the segment between $x$ and $y_{\bar{\lambda}}$. So

$$
F\left(y_{\bar{\lambda}}\right)=F(x)+\bar{\lambda}\left\|(\nabla F)_{x}\right\|+\frac{\bar{\lambda}^{2}}{2\left\|(\nabla F)_{x}\right\|^{2}}(\nabla F)_{x}^{T}(H F)_{\bar{x}}(\nabla F)_{x} .
$$

By the Cauchy-Schwarz inequality we obtain

$$
F\left(y_{\bar{\lambda}}\right) \geq F(x)+\bar{\lambda}\left\|(\nabla F)_{x}\right\|-\frac{\bar{\lambda}^{2}}{2\left\|(\nabla F)_{x}\right\|}\left\|(H F)_{\bar{x}}(\nabla F)_{x}\right\|
$$

and

$$
F\left(y_{\bar{\lambda}}\right) \leq F(x)+\bar{\lambda}\left\|(\nabla F)_{x}\right\|+\frac{\bar{\lambda}^{2}}{2\left\|(\nabla F)_{x}\right\|}\left\|(H F)_{\bar{x}}(\nabla F)_{x}\right\| .
$$

Since $\left\|(H F)_{\bar{x}}(\nabla F)_{x}\right\| \leq\left\|(H F)_{\bar{x}}\right\|\left\|(\nabla F)_{x}\right\|$, we have

$$
F(x)+\bar{\lambda}\left\|(\nabla F)_{x}\right\|-\frac{\bar{\lambda}^{2}}{2}\left\|(H F)_{\bar{x}}\right\| \leq F\left(y_{\bar{\lambda}}\right) \leq F(x)+\bar{\lambda}\left\|(\nabla F)_{x}\right\|+\frac{\bar{\lambda}^{2}}{2}\left\|(H F)_{\bar{x}}\right\| .
$$


Since $\bar{x} \in E$ and $M_{H}<\infty$ we obtain

$$
F(x)+\bar{\lambda}\left\|(\nabla F)_{x}\right\|-\frac{\bar{\lambda}^{2}}{2} M_{H} \leq F\left(y_{\bar{\lambda}}\right) \leq F(x)+\bar{\lambda}\left\|(\nabla F)_{x}\right\|+\frac{\bar{\lambda}^{2}}{2} M_{H}
$$

For $0<\bar{\lambda}<\zeta$, we have from the left side of (8.1)

$$
F\left(y_{\bar{\lambda}}\right) \geq F(x)+\bar{\lambda}\left\|(\nabla F)_{x}\right\|-\frac{\bar{\lambda}^{2}}{2} M_{H} \geq F(x)+\bar{\lambda} m^{\nabla}-\frac{\bar{\lambda}^{2}}{2} M_{H} .
$$

We assume now that $M_{H}>0$ (the case $M_{H}=0$ is trivial).

For $x \in\left\{z \in[0, T]^{2}:|F(z)-c| \leq r\right\}$ and $0<\bar{\lambda}<\zeta \wedge \frac{m^{\nabla}}{M_{H}}$ we have then

$$
F\left(y_{\bar{\lambda}}\right) \geq F(x)+\frac{\bar{\lambda}}{2} m^{\nabla}
$$

Similarly using the right side of (8.1) we obtain for $0<\bar{\lambda}<\zeta \wedge \frac{m^{\nabla}}{M_{H}}$ and $x \in\left\{z \in[0, T]^{2}:|F(z)-c| \leq r\right\}$,

$$
F\left(y_{-\bar{\lambda}}\right) \leq F(x)-\frac{\bar{\lambda}}{2} m^{\nabla} .
$$

Define

$$
\gamma=\left(\frac{m^{\nabla}}{4}\left(\zeta \wedge \frac{m^{\nabla}}{M_{H}}\right)\right) \wedge r>0 .
$$

Suppose that $t=c+\varepsilon, 0<\varepsilon \leq \gamma$. Let $x \in[0, T]^{2}$ such that $F(x)=t=c+\varepsilon$ then $x \in\left\{z \in[0, T]^{2}: \mid F(z)-\right.$ $c \mid \leq r\}$. Take now

$$
0<\bar{\lambda}=\frac{2 \varepsilon}{m^{\nabla}}<\zeta \wedge \frac{m^{\nabla}}{M_{H}}
$$

We obtain from $(8.3)$

$$
F\left(y_{-\bar{\lambda}}\right) \leq F(x)-\frac{\bar{\lambda}}{2} m^{\nabla}=c+\varepsilon-\varepsilon=c .
$$

From the continuity property of $F$, we deduce that there exists $y$ between $x$ and $y_{-\bar{\lambda}}$ such that $F(y)=c$ and we have

So we have proved that

$$
\|x-y\| \leq\left\|x-y_{-\bar{\lambda}}\right\|=|\bar{\lambda}|=\frac{2 \varepsilon}{m^{\nabla}}=\frac{2}{m^{\nabla}}|t-c| .
$$

$$
\sup _{x \in\{F=t\}^{T}} d\left(x,\{F=c\}^{T}\right) \leq \frac{2}{m^{\nabla}}|t-c| .
$$

Similarly, take $x \in[0, T]^{2}$ such that $F(x)=c$ and use (8.2) to obtain

$$
\sup _{x \in\{F=c\}^{T}} d\left(x,\{F=t\}^{T}\right) \leq \frac{2}{m^{\nabla}}|t-c| .
$$

The proof in case $t<c$ is completely analogous. So $F$ satisfies Assumption $\mathbf{H}$ (see Sect. 2) with $A=\frac{2}{m^{\nabla}}$.

Proof of Theorem 3.1. Under assumptions of Theorem 3.1, we can always take $T_{1}>0$ such that for all $t$ : $|t-c| \leq r, \partial L(t)^{T_{1}} \neq \emptyset$.

Then for each $n$, for all $t:|t-c| \leq r, \partial L(t)^{T_{n}}$ is a non-empty (and compact) set on $\mathbb{R}_{+}^{2}$.

In each $\left[0, T_{n}\right]^{2}$, from Proposition 2.1, Assumption $\mathbf{H}$ (Sect. 2) is satisfied with

$$
\gamma=\left(\frac{m^{\nabla}}{4}\left(\zeta \wedge \frac{m^{\nabla}}{M_{H}}\right)\right) \wedge r>0 \quad \text { and } \quad A=\frac{2}{m^{\nabla}} .
$$

First we have to find a bound for $\sup _{x \in \partial L(c)^{T_{n}}} d\left(x, \partial L_{n}(c)^{T_{n}}\right)$. 
Take $x \in \partial L(c)^{T_{n}}$ and define $\varepsilon_{n}=2\left\|F-F_{n}\right\|_{\infty}^{T_{n}}$. Using $\left\|F-F_{n}\right\|_{\infty} \rightarrow 0$, a.s., for $n \rightarrow \infty$, then $\varepsilon_{n} \rightarrow 0$, a.s., for $n \rightarrow \infty$. So with probability one there exists $n_{0}$ such that $\forall n \geq n_{0}, \varepsilon_{n} \leq \gamma$.

Since for all $t:|t-c| \leq r \partial L(t)^{T_{n}} \neq \emptyset$, from Assumption $\mathbf{H}$, there exist $u_{n} \equiv u_{x}^{\varepsilon_{n}}$ and $l_{n} \equiv l_{x}^{\varepsilon_{n}}$ in $\left[0, T_{n}\right]^{2}$ such that

$$
\begin{aligned}
F\left(u_{n}\right)=c+\varepsilon_{n} ; & d\left(x, u_{n}\right) \leq A \varepsilon_{n} \\
F\left(l_{n}\right)=c-\varepsilon_{n} ; & d\left(x, l_{n}\right) \leq A \varepsilon_{n} .
\end{aligned}
$$

Suppose now $\left\|F-F_{n}\right\|_{\infty}^{T_{n}}>0$ (the other case is a trivial one). In this case

$$
F_{n}\left(u_{n}\right)=c+\varepsilon_{n}+F_{n}\left(u_{n}\right)-F\left(u_{n}\right) \geq c+\varepsilon_{n}-\left\|F-F_{n}\right\|_{\infty}^{T_{n}}=c+2\left\|F-F_{n}\right\|_{\infty}^{T_{n}}-\left\|F-F_{n}\right\|_{\infty}^{T_{n}}>c,
$$

and in a similar way we can prove that $F_{n}\left(l_{n}\right)<c$.

As $F_{n}\left(l_{n}\right)<c$ and $F_{n}\left(u_{n}\right)>c$, with $u_{n}$ and $l_{n}$ in $\left[0, T_{n}\right]^{2}$, there exists $z_{n} \in \partial L_{n}(c)^{T_{n}} \cap B\left(u_{n}, d\left(u_{n}, l_{n}\right)\right)$ with $d\left(z_{n}, x\right) \leq d\left(z_{n}, u_{n}\right)+d\left(u_{n}, x\right) \leq d\left(u_{n}, l_{n}\right)+d\left(u_{n}, x\right) \leq d\left(u_{n}, x\right)+d\left(x, l_{n}\right)+d\left(u_{n}, x\right) \leq 3 A \varepsilon_{n}=6 A\left\|F-F_{n}\right\|_{\infty}^{T_{n}}$.

Hence, for $n \geq n_{0}$

$$
\sup _{x \in \partial L(c)^{T_{n}}} d\left(x, \partial L_{n}(c)^{T_{n}}\right) \leq 6 A\left\|F-F_{n}\right\|_{\infty}^{T_{n}} .
$$

Let us now bound $\sup _{x \in \partial L_{n}(c)^{T_{n}}} d\left(x, \partial L(c)^{T_{n}}\right)$.

Take $x \in \partial L_{n}(c)^{T_{n}}$. From the a.s. continuity of $F_{n}$ we obtain $F_{n}(x)=c$, a.s., so

$$
|F(x)-c| \leq\left|F(x)-F_{n}(x)\right| \leq\left\|F-F_{n}\right\|_{\infty}^{T_{n}} \leq \varepsilon_{n}, \quad \text { a.s. }
$$

Remember that $\forall n \geq n_{0}, \varepsilon_{n} \leq \gamma$, a.s. Then from Assumption $\mathbf{H} d\left(x, \partial L(c)^{T_{n}}\right) \leq A|F(x)-c| \leq A\left\|F-F_{n}\right\|_{\infty}^{T_{n}}$. We can conclude that with probability one, for $n \geq n_{0}$

$$
\sup _{x \in \partial L_{n}(c)^{T_{n}}} d\left(x, \partial L(c)^{T_{n}}\right) \leq A\left\|F-F_{n}\right\|_{\infty}^{T_{n}} .
$$

We obtain for $n \geq n_{0}, d_{H}\left(\partial L(c)^{T_{n}}, \partial L_{n}(c)^{T_{n}}\right) \leq 6 A\left\|F-F_{n}\right\|_{\infty}^{T_{n}}$, then

$$
d_{H}\left(\partial L(c)^{T_{n}}, \partial L_{n}(c)^{T_{n}}\right)=O\left(\left\|F-F_{n}\right\|_{\infty}^{T_{n}}\right), \quad \text { a.s. }
$$

Hence the result.

Proof of Theorem 4.1. Under assumptions of Theorem 4.1, we can always take $T_{1}>0$ such that for all $t$ : $|t-c| \leq r, \partial L(t)^{T_{1}} \neq \emptyset$. Then for each $n$, for all $t:|t-c| \leq r, \partial L(t)^{T_{n}}$ is a non-empty (and compact) set on $\mathbb{R}_{+}^{2}$.

We consider a positive sequence $\varepsilon_{n}$ such that $\varepsilon_{n} \underset{n \rightarrow \infty}{\rightarrow} 0$. For each $n \geq 1$ the random sets $L(c)^{T_{n}} \triangle L_{n}(c)^{T_{n}}$, $Q_{\varepsilon_{n}}=\left\{x \in\left[0, T_{n}\right]^{2}:\left|F-F_{n}\right| \leq \varepsilon_{n}\right\}$ and $\widetilde{Q}_{\varepsilon_{n}}=\left\{x \in\left[0, T_{n}\right]^{2}:\left|F-F_{n}\right|>\varepsilon_{n}\right\}$ are measurable and

$$
\lambda\left(L(c)^{T_{n}} \triangle L_{n}(c)^{T_{n}}\right)=\lambda\left(L(c)^{T_{n}} \triangle L_{n}(c)^{T_{n}} \cap Q_{\varepsilon_{n}}\right)+\lambda\left(L(c)^{T_{n}} \triangle L_{n}(c)^{T_{n}} \cap \widetilde{Q}_{\varepsilon_{n}}\right) .
$$

Since $L(c)^{T_{n}} \triangle L_{n}(c)^{T_{n}} \cap Q_{\varepsilon_{n}} \subset\left\{x \in\left[0, T_{n}\right]^{2}: c-\varepsilon_{n} \leq F<c+\varepsilon_{n}\right\}$ we obtain

$$
\lambda\left(L(c)^{T_{n}} \triangle L_{n}(c)^{T_{n}}\right) \leq \lambda\left(\left\{x \in\left[0, T_{n}\right]^{2}: c-\varepsilon_{n} \leq F<c+\varepsilon_{n}\right\}\right)+\lambda\left(\widetilde{Q}_{\varepsilon_{n}}\right) .
$$

From Assumption $\mathbf{H}$ (Sect. 2) and Proposition 2.1, if $2 \varepsilon_{n} \leq \gamma$ then

$$
d_{H}\left(\partial L\left(c+\varepsilon_{n}\right)^{T_{n}}, \partial L\left(c-\varepsilon_{n}\right)^{T_{n}}\right) \leq 2 \varepsilon_{n} A .
$$


So we can write

$$
\lambda\left(\left\{x \in\left[0, T_{n}\right]^{2}: c-\varepsilon_{n} \leq F<c+\varepsilon_{n}\right\}\right) \leq 2 \varepsilon_{n} A 2 T_{n} .
$$

If we now choose

$$
\varepsilon_{n}=o\left(\frac{1}{p_{n} T_{n}}\right)
$$

we obtain that for $n$ large enough $2 \varepsilon_{n} \leq \gamma$ and

$$
p_{n} \lambda\left(\left\{x \in\left[0, T_{n}\right]^{2}: c-\varepsilon_{n} \leq F<c+\varepsilon_{n}\right\}\right) \underset{n \rightarrow \infty}{\rightarrow} 0 .
$$

Let us now prove that $p_{n} \lambda\left(\widetilde{Q}_{\varepsilon_{n}}\right) \underset{n \rightarrow \infty}{\stackrel{\mathbb{P}}{\rightarrow}} 0$. We write

$$
p_{n} \lambda\left(\widetilde{Q}_{\varepsilon_{n}}\right)=p_{n} \int 1_{\left\{x \in\left[0, T_{n}\right]^{2}:\left|F-F_{n}\right|>\varepsilon_{n}\right\}} \lambda(\mathrm{d} x) \leq \frac{p_{n}}{\varepsilon_{n}^{p}} \int_{\left[0, T_{n}\right]^{2}}\left|F-F_{n}\right|^{p} \lambda(\mathrm{d} x) .
$$

Take $\varepsilon_{n}$ such that

$$
\varepsilon_{n}=\left(\frac{p_{n}}{v_{n}}\right)^{\frac{1}{p}}
$$

Then, from Assumption A1, in Section 4, we obtain $p_{n} \lambda\left(\widetilde{Q}_{\varepsilon_{n}}\right) \underset{n \rightarrow \infty}{\stackrel{\mathbb{P}}{\rightarrow}} 0$. As $p_{n}=o\left(v_{n}^{1 / p+1} / T_{n}^{p / p+1}\right)$ we can choose $\varepsilon_{n}$ that satisfies (8.5) and (8.7). Hence the result.

Proof of Theorem 5.3. We only prove the result for the first coordinate of $\operatorname{CTE}_{\alpha}(X, Y)$ (the proof is similar for the second one). First we remark that, in the following, probabilities involving events which depend on $L_{n}(\alpha)^{T_{n}}$, are conditional probabilities to the sample $\left(X_{i}, Y_{i}\right)$, for $i=1, \ldots n$. We will denote $\mathbb{P}\left[(X, Y) \in L_{n}(\alpha)^{T_{n}}\right]$ purely for reasons of notational convenience.

We introduce these two preliminary results (Lems. 8.1 and 8.2):

Lemma 8.1. Under Assumption A2, Assumptions of Theorem 4.1 and with the notation of Theorem 4.1, it holds that

$$
p_{n}^{\frac{r}{2(1+r)}}\left|\mathbb{E}\left[X \mid(X, Y) \in L(\alpha)^{T_{n}}\right]-\mathbb{E}\left[X \mid(X, Y) \in L_{n}(\alpha)^{T_{n}}\right]\right| \underset{n \rightarrow \infty}{\stackrel{\mathbb{P}}{\rightarrow}} 0
$$

with $r>0$ satisfying Assumption A2.

Proof of Lemma 8.1. From Assumption A2 and Theorem 4.1 we obtain

$$
p_{n}^{\frac{r}{2(1+r)}}\left|\mathbb{P}\left[(X, Y) \in L(\alpha)^{T_{n}} \triangle L_{n}(\alpha)^{T_{n}}\right]\right| \leq p_{n}^{\frac{r}{2(1+r)}} d_{\lambda}\left(L(\alpha)^{T_{n}}, L_{n}(\alpha)^{T_{n}}\right)^{\frac{r}{1+r}}\|f\|_{1+r} \underset{n \rightarrow \infty}{\stackrel{\mathbb{P}}{\rightarrow}} 0 .
$$

As a straightforward consequence we find

$$
p_{n}^{\frac{r}{2(1+r)}}\left|\mathbb{P}\left[(X, Y) \in L(\alpha)^{T_{n}}\right]-\mathbb{P}\left[(X, Y) \in L_{n}(\alpha)^{T_{n}}\right]\right| \underset{n \rightarrow \infty}{\stackrel{\mathbb{P}}{\rightarrow}} 0 .
$$

Using Assumption A2 we also obtain

$$
\begin{array}{r}
p_{n}^{\frac{r}{2(1+r)} \mid}\left|\int_{L(\alpha)^{T_{n}}} x f_{X, Y}(x, y) \lambda(\mathrm{d} x \mathrm{~d} y)-\int_{L_{n}(\alpha)^{T_{n}}} x f_{X, Y}(x, y) \lambda(\mathrm{d} x \mathrm{~d} y)\right| \\
\leq p_{n}^{\frac{r}{2(1+r)}} \mathbb{E}\left[X^{2}\right]^{\frac{1}{2}} d_{\lambda}\left(L(\alpha)^{T_{n}}, L_{n}(\alpha)^{T_{n}}\right)^{\frac{r}{2(1+r)}}\|f\|_{1+r}^{\frac{1}{2}} \underset{n \rightarrow \infty}{\stackrel{\mathbb{P}}{\rightarrow} 0 .}
\end{array}
$$


Then

$$
\begin{aligned}
& p_{n}^{\frac{r}{2(1+r)}}\left|\mathbb{E}\left[X \mid(X, Y) \in L(\alpha)^{T_{n}}\right]-\mathbb{E}\left[X \mid(X, Y) \in L_{n}(\alpha)^{T_{n}}\right]\right| \\
&=p_{n}^{\frac{r}{2(1+r)}} \mid \int_{L(\alpha)^{T_{n}}} x f_{X, Y}(x, y) \lambda(\mathrm{d} x \mathrm{~d} y) \mathbb{P}\left[(X, Y) \in L(\alpha)^{T_{n}}\right]^{-1} \\
&-\int_{L_{n}(\alpha)^{T_{n}}} x f_{X, Y}(x, y) \lambda(\mathrm{d} x \mathrm{~d} y) \mathbb{P}\left[(X, Y) \in L_{n}(\alpha)^{T_{n}}\right]^{-1} \mid \\
& \leq \frac{p_{n}^{\frac{r}{2(1+r)}}}{\mathbb{P}\left[(X, Y) \in L(\alpha)^{T_{n}}\right] \mathbb{P}\left[(X, Y) \in L_{n}(\alpha)^{T_{n}}\right]}\left(\mathbb{P}\left[(X, Y) \in L(\alpha)^{T_{n}}\right]\right. \\
& \times\left|\int_{L(\alpha)^{T_{n}}} x f_{X, Y}(x, y) \lambda(\mathrm{d} x \mathrm{~d} y)-\int_{L_{n}(\alpha)^{T_{n}}} x f_{X, Y}(x, y) \lambda(\mathrm{d} x \mathrm{~d} y)\right| \\
&\left.+\int_{L(\alpha)^{T_{n}}} x f_{X, Y}(x, y) \lambda(\mathrm{d} x \mathrm{~d} y)\left|\mathbb{P}\left[(X, Y) \in L(\alpha)^{T_{n}}\right]-\mathbb{P}\left[(X, Y) \in L_{n}(\alpha)^{T_{n}}\right]\right|\right) .
\end{aligned}
$$

Using (8.8)-(8.9) we obtain that (8.10) converges to zero in probability, for $n \rightarrow \infty$. Hence the result.

Lemma 8.2. Under Assumption A2, Assumptions of Theorem 4.1 and with the notation of Theorem 4.1, it holds that

$$
a_{n}\left|\mathbb{E}\left[X \mid(X, Y) \in L_{n}(\alpha)^{T_{n}}\right]-\frac{\sum_{i=1}^{n} X_{i} 1_{\left\{\left(X_{i}, Y_{i}\right) \in L_{n}(\alpha)^{T_{n}}\right\}}}{\sum_{i=1}^{n} 1_{\left\{\left(X_{i}, Y_{i}\right) \in L_{n}(\alpha)^{T_{n}}\right\}}}\right| \underset{n \rightarrow \infty}{\stackrel{\mathbb{P}}{\rightarrow}} 0,
$$

for $a_{n}=o(\sqrt{n})$.

Proof of Lemma 8.2. We can write

$$
\begin{aligned}
& a_{n}\left|\mathbb{E}\left[X \mid(X, Y) \in L_{n}(\alpha)^{T_{n}}\right]-\frac{\sum_{i=1}^{n} X_{i} 1_{\left\{\left(X_{i}, Y_{i}\right) \in L_{n}(\alpha)^{T_{n}}\right\}}}{\sum_{i=1}^{n} 1_{\left\{\left(X_{i}, Y_{i}\right) \in L_{n}(\alpha)^{T_{n}}\right\}}}\right| \\
& =a_{n}\left|\frac{\int_{L_{n}(\alpha)^{T_{n}}} x f_{X, Y}(x, y) \lambda(\mathrm{d} x \mathrm{~d} y)}{\mathbb{P}\left[(X, Y) \in L_{n}(\alpha)^{T_{n}}\right]}-\frac{\sum_{i=1}^{n} X_{i} 1_{\left\{\left(X_{i}, Y_{i}\right) \in L_{n}(\alpha)^{T_{n}}\right\}}}{\sum_{i=1}^{n} 1_{\left\{\left(X_{i}, Y_{i}\right) \in L_{n}(\alpha)^{T_{n}}\right\}}}\right| .
\end{aligned}
$$

Under assumptions of Lemma 8.2, from the central limit theorem for triangular arrays (e.g. Thm. 27.2 in Billingsley [6]) we obtain

$$
\begin{aligned}
& a_{n} \mid \mathbb{P}\left[(X, Y) \in L_{n}(\alpha)^{T_{n}}\right]-\frac{1}{n} \sum_{i=1}^{n} 1_{\left\{\left(X_{i}, Y_{i}\right) \in L_{n}(\alpha)^{\left.T_{n}\right\}} \mid\right.} \underset{n \rightarrow \infty}{\stackrel{\mathbb{P}}{\rightarrow}} 0, \\
& a_{n} \mid \int_{L_{n}(\alpha)^{T_{n}}} x f_{X, Y}(x, y) \lambda(\mathrm{d} x \mathrm{~d} y)-\frac{1}{n} \sum_{i=1}^{n} X_{i} 1_{\left\{\left(X_{i}, Y_{i}\right) \in L_{n}(\alpha)^{\left.T_{n}\right\}} \mid\right.} \underset{n \rightarrow \infty}{\stackrel{\mathbb{P}}{\rightarrow} 0 .}
\end{aligned}
$$

Hence the result. 
Then to prove Theorem 5.3 we can write (5.4) as

$$
\begin{aligned}
& \beta_{n} \mid \mathbb{E}\left[X \mid(X, Y) \in L(\alpha)^{T_{n}}\right]-\frac{\sum_{i=1}^{n} X_{i} 1_{\left\{\left(X_{i}, Y_{i}\right) \in L_{n}(\alpha)^{T_{n}}\right\}} \mid}{\sum_{i=1}^{n} 1_{\left\{\left(X_{i}, Y_{i}\right) \in L_{n}(\alpha)^{T_{n}}\right\}} \mid} \\
& \leq \beta_{n}\left|\mathbb{E}\left[X \mid(X, Y) \in L(\alpha)^{T_{n}}\right]-\mathbb{E}\left[X \mid(X, Y) \in L_{n}(\alpha)^{T_{n}}\right]\right| \\
& +\beta_{n}\left|\mathbb{E}\left[X \mid(X, Y) \in L_{n}(\alpha)^{T_{n}}\right]-\frac{\sum_{i=1}^{n} X_{i} 1_{\left\{\left(X_{i}, Y_{i}\right) \in L_{n}(\alpha)^{T_{n}}\right\}}}{\sum_{i=1}^{n} 1_{\left\{\left(X_{i}, Y_{i}\right) \in L_{n}(\alpha)^{T_{n}}\right\}}}\right| .
\end{aligned}
$$

The result is a straightforward application of Lemmas 8.1 and 8.2.

Acknowledgements. The authors thank two anonymous referees for relevant remarks and constructive comments on a previous version of the paper. The authors thank Alberto Rodríguez-Casal for his fruitful comments. They thank also Stéphane Loisel and Ragnar Norberg for helpful discussions. This work has been partially supported by the French research national agency (ANR) under the reference ANR-08BLAN-0314-01. The fourth author thanks the BNP Paribas Cardif Chair "Management de la modélisation" for financial support. The views expressed in this document are the author's own and do not necessarily reflect those endorsed by BNP Paribas Cardif.

\section{REFERENCES}

[1] P. Artzner, F. Delbaen, J.-M. Eber and D. Heath, Coherent measures of risk. Math. Finance 9 (1999) $203-228$.

[2] A. Baíllo, Total error in a plug-in estimator of level sets. Statist. Probab. Lett. 65 (2003) 411-417.

[3] A. Baíllo, J.A. Cuesta-Albertos and A. Cuevas, Convergence rates in nonparametric estimation of level sets. Statist. Probab. Lett. 53 (2001) 27-35.

[4] F. Belzunce, A. Castaño, A. Olvera-Cervantes and A. Suárez-Llorens, Quantile curves and dependence structure for bivariate distributions. Comput. Stat. Data Anal. 51 (2007) 5112-5129.

[5] G. Biau, B. Cadre and B. Pelletier, A graph-based estimator of the number of clusters. ESAIM: PS 11 (2007) 272-280.

[6] P. Billingsley, Probability and measure. Wiley Series in Probability and Mathematical Statistics, 3th edition, John Wiley \& Sons Inc., A Wiley-Interscience Publication, New York (1995).

[7] B. Cadre, Kernel estimation of density level sets. J. Multivar. Anal. 97 (2006) 999-1023.

[8] J. Cai and H. Li, Conditional tail expectations for multivariate phase-type distributions. J. Appl. Probab. 42 (2005) 810-825.

[9] L. Cavalier, Nonparametric estimation of regression level sets. Statistics (Berl. DDR) 29 (1997) 131-160.

[10] Y.P. Chaubey and P.K. Sen, Smooth estimation of multivariate survival and density functions. J. Statist. Plann. Inference 103 (2002) 361-376; C. R. Rao 80th birthday felicitation volume, Part I.

[11] A. Cuevas and R. Fraiman, A plug-in approach to support estimation. Ann. Stat. 25 (1997) 2300-2312.

[12] A. Cuevas and A. Rodríguez-Casal, On boundary estimation. Adv. Appl. Probab. 36 (2004) 340-354.

[13] A. Cuevas, W. González-Manteiga and A. Rodríguez-Casal, Plug-in estimation of general level sets. Australian ES New Zealand J. Statist. 48 (2006) 7-19.

[14] L. de Haan and X. Huang, Large quantile estimation in a multivariate setting. J. Multivar. Anal. 53 (1995) $247-263$.

[15] S. Dedu and R. Ciumara, Restricted optimal retention in stop-loss reinsurance under VaR and CTE risk measures. Proc. of Rom. Acad. Ser. A 11 (2010) 213-217.

[16] M. Denuit, J. Dhaene, M. Goovaerts and R. Kaas, Actuarial Theory for Dependent Risks. Wiley, (2005).

[17] P. Embrechts and G. Puccetti, Bounds for functions of multivariate risks. J. Multivar. Anal. 97 (2006) $526-547$.

[18] J.M. Fernández-Ponce and A. Suárez-Lloréns, Central regions for bivariate distributions. Austrian J. Stat. 31 (2002) $141-156$.

[19] E.W. Frees and E.A. Valdez, Understanding relationships using copulas. North Amer. Actuar. J. 2 (1998) 1-25.

[20] J.A. Hartigan, Estimation of a convex density contour in two dimensions. J. Amer. Statist. Assoc. 82 (1987) $267-270$.

[21] V.I. Koltchinskii, M-estimation, convexity and quantiles. Ann. Statist. 25 (1997) 435-477.

[22] T. Laloë, Sur Quelques Problèmes d'Apprentissage Supervisé et Non Supervisé. Ph.D. thesis, University Montpellier II (2009).

[23] J.-C. Massé and R. Theodorescu, Halfplane trimming for bivariate distributions. J. Multivar. Anal. 48 (1994) $188-202$. 
[24] G. Nappo and F. Spizzichino, Kendall distributions and level sets in bivariate exchangeable survival models. Inform. Sci. 179 (2009) 2878-2890.

[25] W. Polonik, Measuring mass concentrations and estimating density contour clusters - an excess mass approach. Ann. Stat. 23 (1995) 855-881.

[26] W. Polonik, Minimum volume sets and generalized quantile processes. Stoch. Proc. Appl. 69 (1997) 1-24.

[27] P. Rigollet and R. Vert, Optimal rates for plug-in estimators of density level sets. Bernoulli. 15 (2009) 1154-1178.

[28] A. Rodríguez-Casal. Estimacíon de conjuntos y sus fronteras. Un enfoque geometrico. Ph.D. thesis, University of Santiago de Compostela (2003).

[29] C. Rossi, Sulle curve di livello di una superficie di ripartizione in due variabili; on level curves of two dimensional distribution function. Giornale dell'Istituto Italiano degli Attuari 36 (1973) 87-108.

[30] C. Rossi, Proprietà geometriche delle superficie di ripartizione. Rend. Mat. (6) 9 (1976) 725-736 (1977).

[31] R. Serfling, Quantile functions for multivariate analysis: approaches and applications. Stat. Neerlandica 56 (2002) 214-232 Special issue: Frontier research in theoretical statistics (2000) (Eindhoven).

[32] L. Tibiletti, On a new notion of multidimensional quantile. Metron 51 (1993) 77-83.

[33] A.B. Tsybakov, On nonparametric estimation of density level sets. Ann. Stat. 25 (1997) 948-969. 